\title{
„Die Gesellschaft aber hat Anspruch auf Schutz vor ihnen“ - Der eugenische Diskurs über die Asylierung von Anormalen
}

\author{
Gisep Buchli
}

\begin{abstract}
„Vereinigen wir nun den Begriff der Konstitution mit dem der Norm, so können wir von einer normalen, also zweckmässig beschaffenen Konstitution, bzw. von einer abnormen, also unzweckmässig veranlagten Konstitution sprechen. ${ }^{\text {1 }}$
\end{abstract}

\section{Prolog}

In diesem Aufsatz möchte ich die Diskriminierung von „Behinderten“ historisch darstellen. Dazu versuche ich die Frage zu beantworten: Wie, wann und vor allem wieso kam es zur Erfindung von „Heimen“ für „Behinderte“?”

Dieser Aufsatz versteht sich als Beitrag zur disability history. Nur bei dem kleinsten Teil der Autoren dieser historischen Spezialdisziplin handelt es sich um Geschichtswissenschaftler, in den allermeisten Fällen sind es Sonder-, Heil- oder Integrationspädagogen oder Hobbyhistoriker, die sich damit beschäftigen: Die disability history hat sich als historisches Teilgebiet noch nicht endgültig etablieren können.

1 Zurukzoglu (1938), S. 16. - Das Zitat im Titel stammt aus Forel (1905), S. 280.

2 „Heime“ für „Behinderte“ sind gerade im deutschsprachigen Raum noch heute massenweise vorhanden. - Am 31. Dezember 1930 - etwa am Zenit der abendländischen Eugenik - erließ Papst Pius XI. die Enzyklika Casti connubii, in der er sich mit deutlichen Worten gegen die "negative“ Eugenik (Verhinderung der Fortpflanzung Erbkranker; u.a. Asylierung) aussprach. Dies ist der Grund, wieso sich die Eugenik in den meisten (konservativ) katholischen Ländern nicht oder bedeutend weniger etablieren konnte. Und somit erklärt sich auch die Tatsache, dass Asyle für Behinderte vorwiegend eine protestantische Angelegenheit waren und sind. Da zudem in den angelsächsischen und skandinavischen Ländern und in den Niederlanden schon seit einiger Zeit mit der Hinwendung zu einer independent-living-Politik Heime abgeschafft wurden, handelt es sich bei der Asylierung lediglich um ein zentraleuropäisches und besonders deutschsprachiges Relikt aus der Eugenik. 
Im Anschluss an Foucault nahm der Ethnologe Erving Goffman seit den 1950er Jahren eine sog. Mikroperspektive ein: Mittels teilnehmender Beobachtung stellte er das Funktionieren der internen Abläufe in einer psychiatrischen Großklinik (in Washington D. C.) dar und wies dabei auf die Beziehungen zwischen institutioneller Ordnung und Formen von Zwang hin. 1961 veröffentlichte er seine Erkenntnisse aus dem Aufenthalt im St. Elizabeths Hospital unter dem Titel Asylums: Essays on the Social Situation of Mental Patients and Other Inmates. Darin beschreibt Goffman das Leben in einer „totalen Institution" und vergleicht die Situation der darin Lebenden mit jener in Gefängnissen, Klöstern oder Internaten. Das Asyl bzw. die Institution bezeichnet er deshalb als total, weil sie die sozialen Kontakte der Insassen sowie deren Handlungsspielräume systematisch beschränkt, die Patienten in jeder Hinsicht von den Mitarbeitern unterscheidet und einer umfassenden institutionellen Regulierung unterwirft, die vor allem der Institution und dem Personal nützt. Im Mittelpunkt seiner Analyse steht die These, dass die Institutionen nicht den Interessen der Patienten dienen, sondern im Gegenteil diese für ihre eigenen Interessen brauchen. ${ }^{3}$

Die Suche nach dem Ursprung von Heimen führt ins letzte Drittel des 19. Jahrhunderts. Der Sozialdarwinismus und besonders die daraus folgende Eugenik beruhten auf einem folgenschweren Irrtum: nämlich auf der in der Mitte des 19. Jahrhunderts entwickelten Degenerationstheorie.

Überdeutlich ist bei der Geschichte der Asylierung die stets präsente Sexualisierung. Erkennbar wird sie nicht nur in George Beards Neurasthenie ${ }^{4}$ oder Charcots Hysterie, ${ }^{5}$ sondern in der gesamten psychiatrischen Literatur zur Nervosität begegnet sie uns auf Schritt und Tritt, am deutlichsten vielleicht in Krafft-Ebings Pathologia Sexualis oder in der Schrift Sexuelle Frage des Zürcher Professors und ersten Direktors vom Burghölzli, August Forel.

Bei der Asylidee handelte es sich im Wesentlichen um die Verhinderung der Fortpflanzung, um eine Sexualkontrolle. Der gesamte Heimdiskurs drehte sich um diese Frage, und nicht etwa darum, ob es günstiger sei, Anormale massenweise zu pflegen oder nicht.

Eminent wichtig für das Verstehen der dramatischen Geschichte der „Behinderten“, die ohne Zweifel ihren (praktischen) Höhepunkt in Nazi-Deutschland erreichte, ${ }^{6}$ ist der Umstand, dass die essentielle Unterscheidung zwischen geistiger, psychischer und körperlicher Behinderung sowie Sinnesbehinderung $^{7}$ bis vor kurzem nicht existierte. ${ }^{8}$

Die Bezeichnung „Behinderte“ ist keineswegs alt: Erst seit den 1930er Jahren existiert sie. In der Schweiz fand diese literarisch erst 1942 ihren Gebrauch:

3 Goffman (1961).

4 Vgl. Roelcke (1999), S. 112-121.

5 Vgl. Didi-Hubermann (1997).

6 Vgl. Trus (1995); Klee (1989).

7 Spätestens seit Griesinger (1845) hatte sich in der Psychiatrie das Paradigma „Geisteskrankheiten sind Gehirnkrankheiten“ eingebürgert. - Vgl. Kolle (1956), S. 115-127.

8 Nach Dieter Mattner wird die „Gruppe der Behinderten üblicherweise in 7 Untergruppen unterteilt: Körperbehinderte, Sinnesbehinderte, Geistigbehinderte, Sprachbehinderte, Lernbehinderte, Verhaltensgestörte bzw. Verhaltensbehinderte, Schwerst(mehrfach)behinderte“. - Vgl. Mattner (2000), S. 9.

In der Schweiz existiert sie erst seit der letzten Verfassungsreform (1.1.2000) offiziell. - Vgl. Thüerer (2001); insbes. darin Erwin Murer, S. 967-978. 
Erst jetzt wurde der Begriff „Anormale“ durch jene noch heute gebrauchte Bezeichnung „Behinderte“ ersetzt. Der neue Ausdruck erscheint in der Schweiz erstmals in der Dissertation des Berner Juristen Oswald Rohrer. ${ }^{9}$

Unter „Behinderte“ verstand er: „Blinde, Taubstumme, Schwerhörige, Epileptische, Geistesschwache, Krüppelhafte, Invalide, Schwererziehbare und Psychopathen." 10

Beim neuen Begriff handelt es sich lediglich um eine sprachliche Abänderung; was man darunter verstand, blieb auch nach der begrifflichen Metamorphose dasselbe: Menschen, die anders als die „Normalen“ waren: eben „Anormale“. Trotz der Einführung des Begriffs durch die Pro Infirmis, die aber weiterhin ,alle Geisteskranken“11 von jeglicher Unterstützung ausschloss, blieben andere Bezeichnungen bis Ende des 20. Jahrhunderts bestehen.

Erst seit dem Fin de Siècle unterschied man Menschen in gesund und krank bzw. normal und anormal. ${ }^{12}$ Obwohl die Diskriminierung einzelner Behinderungen möglicherweise so alt wie die Menschheit ist, ${ }^{13}$ kann von einer systematischen Verfolgung und Segregation erst seit dem späten 19. Jahrhundert, seit der späten Aufklärung bzw. als Folge der Industrialisierung, die Rede sein.

9 Diese Dissertation erschien als Beiheft im Druck 1945 in: Zurukzoglu: Gesundheitspflege der Gegenwart. Sie ist im Wesentlichen eine Werbeschrift für die Pro Infirmis.

Die Bezeichnung „behindert“ taucht erstmals anlässlich einer statistischen Erfassung jugendlicher Krüppel in Preußen im Jahre 1906 auf. Dabei wurde der Krüppel als „ein in dem Gebrauch seines Rumpfes oder seiner Gliedmassen behinderter Kranker" definiert. - Vgl. Waldschmidt (2006). - Der erste mir bekannte Gebrauch der neuen Terminologie - ebenfalls noch als Adjektiv gebraucht - datiert aus dem Jahr 1875 (in einer Beschreibung von Hermann dem Lahmen von Friedrich Wilhelm von Giesebrecht). 1919 übernahm eine Krüppelvereinigung, der Otto-Perl-Bund in Berlin, diesen Begriff, der somit zum Zusammenschluss körperlich Behinderter wurde. Offiziell blieb jedoch der Krüppelbegriff weiterhin bestehen. Das nationalsozialistische Gesetz zur Vereinheitlichung des Gesundheitswesens von 1934 benutzte erstmals den Begriff "körperlich Behinderte“ als Sammelbezeichnung. Vgl. Waldschmidt (2006), S. 37. Kurz darauf, im Jahr 1935, wurde in der Schweiz die 1919 gegründete Vereinigung für Anormale zur Pro Infirmis - eine Gesellschaft, ohne die dort noch heute Behindertenpolitik undenkbar ist und wollte von nun an nicht mehr den Anormalen helfen, sondern „allen Behinderten“.

10 Rohrer (1945), S. 86.

11 Rohrer (1945), S. 21.

12 Vgl. Kaiser (1927).

13 Berichte über Misshandlungen oder Tötungen von Menschen mit einer spezifischen Behinderung finden sich immer wieder. So die folgende Geschichte, die sich 1565 in Schmitz zutrug „Die Geburt habe kein Haupt gehabt, in seiner Brust habe sich an der linken Achsel der Mund, an der rechten ein Ohr befunden. An Händen und Füssen habe es froschartige Extremitäten gehabt. Der Leib sei braun wie eine Leber gewesen und habe gezittert wie Gallert, da keine Knochen in ihm gewesen seien. Als das Kind, nachdem es vorher von der Gemeinde vor der Kirche betrachtet worden war, auf dem Kirchhof an der Stelle für die Ungetauften begraben worden war, habe die Mutter immer wieder gedrängt, es solle ausgegraben und verbrannt werden. Schließlich bekannte sie, dass es das Produkt von Unzucht mit dem Teufel sei, der des Nachts in Gestalt ihres Mannes zu ihr gekommen sei. Deshalb bat sie, man solle die Geburt dem Teufel überlassen und sie vor seinen weiteren Angriffen schützen. Die Geburt wurde wieder ausgegraben und verbrannt, wobei dies trotz großer Mengen Holzes nicht gelingen wollte. Selbst die Windeln seien nass geblieben (!). Schließlich sei die Geburt zerhackt worden, worauf das Verbrennen gelungen sei. In der Nacht darauf sei vor dem Haus der Frau lautes Kreischen und Heulen aufgetreten, das schließlich nach mehrmaligen Beten und Anrufen Gottes verstummt sei.“ - Vgl. Ewinkel (1995), S. 191.

Ähnliche Beispiele werden in großer Zahl und durch alle Zeiten, von den Spartanern, den Römern über das Mittelalter bis hin zur Moderne, von anderen Autoren genannt. - Vgl. Fandey (1990), Wolfisberger (1995) und (2002), Mürner (1996), Frehe (2004), Mattner (2000) oder Kaba (2006). Diese Autoren begehen aber alle einen ungeheuerlichen Anachronismus: Behinderte, von denen sie alle sprachen, gab es vor dem 19. Jahrhundert nicht. Mit Vorliebe werden von zahlreichen Autoren der disability history vermeintliche Aussprüche von Martin Luther ohne Quellenangabe! - zitiert. Dazu ist Folgendes anzumerken: Ob Luther sich jemals zu den „Wechselbälgen“, die man „ersäufen soll“ geäußert hat, ist sehr ungewiss. Jedenfalls fehlt von ihm jegliche schriftliche Mitteilung zu diesem Thema: Seine sog. „Tischreden“ wurden postum publiziert. - Vgl. Haustein (1990). 


\section{Asyle als eugenische Maßnahmen}

1938 meinte der erste Inhaber des Lehrstuhls der Sonderpädagogik in Zürich, Prof. Dr. Heinrich Hanselmann, dass für die „nicht genügend zu schützenden Fälle [...] nur die dauernde Internierung übrig“ bleibe. Er betont, dass sich dabei eine Sterilisierung „,wohl zumeist erübrigt“. Seiner Meinung nach kommt entweder „die Unterbringung in einer Irrenpflegeanstalt in Betracht“, für andere Anormale „müsste vielleicht die geeignete Anstalt“ noch erfunden werden. Denn eine „selbstverständlich geschlechtertrennende Unterbringung“ schließe eine „Fortpflanzung praktisch fast ganz aus“. Die Verhinderung der Fortpflanzung um jeden Preis „,von Geistesschwachen der mittleren und schweren Grade, einer Großzahl ausgesprochen psychopathischer Persönlichkeiten, sowie von Geisteskranken der degenerativen, endogenen Formenkreise“ sei „,vom Standpunkt der Volkswohlfahrtspflege ein dringendes und unausweichliches Erfordernis“. ${ }^{14}$

Der Autor des Zitats war keineswegs ein Anhänger Adolf Hitlers und kein Nazi. Seine Ansicht war zu jener Zeit common sense, nicht nur im Dritten Reich, sondern in ganz Europa und Nordamerika.

Der Berner Privatdozent Stavros Zurukzoglu plädiert in seiner Aufsatzsammlung „Verhütung erbkranken Nachwuchses ${ }^{15}$ von 1938 für die Nachahmung des von den Nazis unmittelbar nach deren Machtergreifung (Januar 1933) eingeführten Sterilisationsgesetzes. ${ }^{16}$ Er meint, dass „als Mittel zur Verhinderung der Fortpflanzung“ von Anormalen neben der Sterilisation „auch die Unterbringung in geschlossenen Anstalten“ in Frage komme. „Der Zustand vieler Erbkranker“ sei nämlich so, „dass eine Dauerversorgung in Anstalten unumgänglich“ sei. Dadurch werde zugleich „auch die Verhütung erbkranken Nachwuchses erreicht“. Und weiter schreibt Zurukzoglu: so sehe man, „dass die Asylierung nicht als Gegensatz zu anderen eugenischen Massnahmen [...] aufzufassen ist“. Und schließlich sei auch die Meinung falsch, „dass durch eine Umgestaltung des Asylierungswesens andere Massnahmen entbehrlich würden." 17

An anderer Stelle versichert er, wenn man „die Bestrebungen der Eugenik sinnvoll in das Gesamtgebiet der hygienischen Massnahmen" einbetten und jenen, die aus irgendwelchen Gründen die Sterilisation ablehnen, „die Handhabe für eine wirksame Eindämmung der Fortpflanzung Erbkranker in die Hand geben“ will, so müsse nach einer Lösung gesucht werden, die nicht bloß auf die Sterilisation allein abzielt, „,sondern die der Verhütung erbkran-

14 Hanselmann, in: Zurukzoglu (1938), S. 93.

15 Diese Aufsatzsammlung entstand 1938 mit finanzieller Unterstützung der noch heute existierenden Julius KlausStiftung für Vererbungsforschung, Sozialanthropologie und Rassenhygiene. Seit den frühen 1960er Jahren nennt sie sich „Julius Klaus-Stiftung für Genetik“.

16 Das am 14. Juli 1933 eingeführte „Gesetz zur Verhütung erbkranken Nachwuchses“ (vgl. Trus [1995], S. 61-65) bestimmte, dass die Sterilisation „auch gegen den Willen des Unfruchtbarzumachenden“ auszuführen sei. - Vgl. Haenel (1982), S. 164.

17 Zurukzoglu (1938), S. 51 f.; vgl. auch: Gustav (1990), S. 3-5. 
ken Nachwuchses dienenden Massnahmen, wie Eheberatung, Asylierung, nachgehende Fürsorge, Bewahrungsheime usw. mit einbezieht. "

Zur selben Frage meinte 1934 Hans Wolfgang Maier, der Nachfolger Eugen Bleulers als Professor und Direktor der Zürcher Psychiatrie und außerdem Präsident des „Zürcher Hülfsverein für Geisteskranke“: ${ }^{19}$ „Das deutsche Vorgehen stellt sicherlich auch für andere Länder einen interessanten Versuch dar. “ ${ }^{20} \mathrm{Er}$ fordert wie die Julius Klaus-Stiftung ${ }^{21}$ jedoch eine gewisse „Freiwilligkeit des operativen Eingriffes“: ${ }^{22} 1942$ befand Maier, dass in Fällen „wo es unerlässlich nötig ist, diesen Weg [die Sterilisation] zu beschreiten [wenigstens] keine Gesetze erlassen werden, die [sie] verbieten. “23

Doch woher kommen diese bizarren Ansichten? Die Beantwortung dieser Frage führt in das späte 19. Jahrhundert, in das sog. Fin de Siècle oder, wie es Joachim Radkau es treffend nennt, ins Zeitalter der Nervosität.

In dieser Zeit der sozialdarwinistischen bzw. morelschen Degenerationsangst entstanden die ersten „Heime für Anormale“. In der Schweiz kam bereits im 17. Jahrhundert die Idee auf, Taubstumme gezielt zu fördern. In die Tat umgesetzt wurde sie jedoch erst 1777 mit der Gründung einer Taubstummenanstalt in Schlieren. Um 1800 wurde in kantonalen Zählungen das Bedürfnis nach einer gezielten Schulung von „Sinnesbehinderten“ nachgewiesen. Durch private und religiös motivierte Initiativen entstanden Anfang des 19. Jahrhunderts weitere Institutionen zur Förderung behinderter Kinder. Den Anstalten für Sinnesbehinderte (Blindenschule in Zürich, 1809) folgten im Verlauf des Jahrhunderts weitere für „geistig“ (Anstalt für kretine Kinder bei Interlaken, 1840) und später auch für „körperlich behinderte“ Kinder (Mathilde-Escher-Heim in Zürich, 1864). „Behinderte“ waren von der allgemeinen Schulpflicht ausgenommen und blieben auf private Förderung angewiesen.

\section{Im Bann des Entartungsparadigmas}

„Nur die [erbliche Veranlagung] einer ganzen Bevölkerung lässt sich umstellen, dadurch, dass wir die Fortpflanzung regeln, die Tüchtigen, Hochwertigen vermehren, die Untüchtigen ausschalten. Zielbewusste Zeugung ist der Weg, der zur Gesundung des Volkes führt. Leider ist die heutige Zeit weit entfernt von diesem Ziel. Zwar entspringt die Zeugung nicht mehr jener naiven Einstellung früherer Zeiten. Das Triebleben kommt unter die Herrschaft des Verstandes, das Sexualleben wird rationalisiert. “24

18 Gustav (1990), S. $50 \mathrm{f}$.

19 Arnold (1992), S. 28.

20 Maier (1934), S. 790.

21 Schmutz (2000), S. 308.

22 Ebd.

23 Maier (1942), S. 50.

24 Guggisberg, in: Zurukzoglu (1938), S. 62. - Guggisberg (1880-1977) führte nach seiner Emeritierung (1950) eine Privatklinik in Bern bis kurz vor seinem Tod. 
Ein zentrales Moment, das zur Entstehung der Behindertensegregation führte, war die im Fin de Siècle entstandene Degenerationsangst. ${ }^{25}$ Die religiös geprägte, in der Mitte des 19. Jahrhunderts vom französischen Psychiater Bénédict Augustin Morel (1809-1873) erfundene ${ }^{26}$ und vom späteren Zionisten, dem deutschsprachigen Max Nordau ${ }^{27}$ rezipierte Degenerationstheorie besagte erstens, dass Entartung im doppelten Sinn vererbt werden könne: sowohl körperlich wie auch moralisch und zweitens, dass Entartung progressiv sei und zwar bis zum Aussterben der betroffenen Familien. Kurzgefasst besagt Morels Theorie Folgendes: Am Anfang treten in einer Familie psychische Abnormitäten und sittliche Verwahrlosung auf. In der folgenden Ceneration schließen sich schwere Neurosen und Alkoholismus an. In der dritten und vierten Generation kommen schwere geistige Störungen, angeborener Schwachsinn und Missbildungen aller Art hinzu. Durch Unfruchtbarkeit stirbt dann die betroffene Familie aus. ${ }^{28}$

Bedeutend an der grundsätzlichen Veränderung des Zivilisationsdiskurses seit der Aufklärung ist, dass auch die moralische, ästhetische und intellektuelle Sphäre dem Urteil der Medizin unterworfen werden, die sie nach den Kriterien von gesund und krank unterscheidet. Damit wird bei Morel und seinen Nachfolgern der gesamte Diskurs um Entartung durch Zivilisation pathologisiert.

Wie seit Anfang des 19. Jahrhunderts in allen vom Positivismus beeinflussten Wissenschaften (in den Naturwissenschaften ebenso wie in der Soziologie und Medizin) modern geworden, wurde jetzt bei allen Zivilisationsphänomenen nur noch zwischen gesund und krank unterschieden. Das Normale war somit gesund, das von der virtuellen Norm abweichende, Anormale hingegen krank. Entartung durch Zivilisation, sei sie physische, moralische oder intellektuelle Entartung, war eine negative Normabweichung und galt als krank. Die Zivilisationskritik Morels an der Entartung durch Zivilisation fand in den positivistisch orientierten Wissenschaften Anklang. Von entscheidender Bedeutung war, dass Entartung als Krankheit begriffen wurde, und zwar als Geisteskrankheit. Die Zivilisationskritik wurde damit zur Sache des Arztes, besonders des Psychiaters, die Phänomene von Entartung, gleich ob intellektuell, moralisch oder physisch, wurden Gegenstand der Pathologie und besonders der Psychopathologie. ${ }^{29}$

„Der Arzt und Schriftsteller Max Nordau kann sich daher mit vollem Recht innerhalb dieses durch Morel initiierten Diskurses zum Zivilisationskritiker geradezu berufen fühlen. Denn

25 Diese Angst wird sehr schön von Emil Kraepelin in einer Metapher beschrieben: „Welches Volk möchte in den pfadlosen Ozean der Zukunft ohne einen Kompass hinausfahren, dessen Schwankungen ihm zeigen, wann es die Richtung auf das Ziel verloren hat und dem Untergange zusteuert?" - Vgl. Kraepelin (1918), S. 201.

26 Morel (1857).

27 Nordau (1896); vgl. dazu Schulte (1997), bes. S. 201-251; Roelcke (1999), S. 80-88.

28 Hermle, in: Brüne/Payk (2004), S. 26. - Zur Entartung meinte Zurukzoglu: „Entartung ist jede Abweichung von der Norm bzw. vom Typus, welche die physiologische Gesundheitsbreite und die korrelative Variabilität der biologischen Vollwertigkeit im Sinne einer somatischen bzw. psychischen Verschlechterung und des Nichtangepaßtseins überschreitet." Vgl. Zurukzoglu (1925), S. 63. Wortwörtlich wiederholt dies derselbe auch 13 lahre später. Vgl. Zurukzoglu (1938), S. 17.

29 Schulte (1997), S. $201 \mathrm{f}$. 
der Arzt, nicht mehr der Philosoph, der Jurist, der Priester oder der Politiker, diagnostiziert die Entartung durch Zivilisation und therapiert sie dann auch. Entartung im weitesten Sinn wird auf diese Weise zum Therapie-Objekt der Medizin, erst in zweiter Linie der Moral, des Rechts oder der Politik. “30

Emil Kraepelin meinte 1918, dass es „klar auf der Hand“ liege, dass „unsere Gesittung der natürlichen Auslese, die nur die Tüchtigsten erhält und zur Fortpflanzung zulässt, wirksam entgegenarbeitet“. Denn „alle die zahlreichen Schöpfungen menschlichen Mitleids, die darauf abzielen, auch das Leben der Kranken, Schwachen, Untauglichen nach Möglichkeit zu erhalten und menschenwürdig zu gestalten“, hätten „ohne Zweifel“ die ärgerliche Konsequenz, „dass sich unserem Nachwuchse dauernd ein breiter Strom minderwertiger Keime beimischt, der eine Verschlechterung der Rasse bedeutet. " Schlussendlich ist es nach Kraepelin so, dass ,je vollkommener uns [...] die Erfüllung unserer Menschenpflicht gegen die Elenden, Verirrten und Hilflosen gelingt, desto nachhaltiger schädigen wir die Kraft unseres Volkstumes. " ${ }^{11}$

Man kann - sicherlich zu Recht - die Frage stellen, ob es denn vor dieser Zeit keine Behinderten gegeben hätte. Ähnlich wie die Frauenbewegung Sex und Gender trennt, so muss man auch hier differenzieren zwischen der naturgegebenen Behinderung einerseits und der Medizinalisierung der Behinderung bzw. der Behinderten andererseits, die vor dem 19. Jahrhundert schlichtweg nicht existierte. Die genannte Zeit ist der Beginn der Pathologisierung der vermeinten Gattung. Nach der Aufklärung, besonders während des 19. Jahrhunderts, wurden verschiedene Krankheiten erfunden, die es vorher nicht gab. Menschen mit einer Behinderung gab es natürlich schon immer und zwar in Relation zur Bevölkerungszahl viel mehr als heute; allein: sie wurden nicht als eine Gruppe identifiziert.

Tatsächlich findet sich im 12. Jahrhundert, im vermeintlich finsteren Mittelalter, ein hervorragendes Beispiel für Antonovskys Salutogenese, ein schwer behinderter Mann, der dank persönlicher Assistenz zu einem der bedeutendsten Gelehrten des Mittelalters wurde: Hermannus Contractus.

\section{Einschub: Hermann von der Reichenau}

Als Paradebeispiel eines Vertreters des Fin de Siècle beschrieb der Münchner Historiker Friedrich Wilhelm von Giesebrecht im Jahr 1875 Hermann den Lahmen wie folgt:

„Verkrüppelt, gichtbrüchig, auch mit der Sprache behindert, ergab er sich, von dem weltlichen Leben ausgeschlossen, ganz dem Studium ... Auf seinen Sessel gebannt, konnte Hermann nur mitteilen, was er in den Büchern fand oder was die Fama inm zuführte; besonders verließ er sich auf die Bücher und hat selbst bei den ihm gleichzeitigen Begebenheiten sie vor allem benutzt. “32

30 Ebd.

31 Kraepelin (1918), S. 196.

32 Zitiert aus Borst (1988a), S. 135. 
Im gleichen Jahr beschreibt der Pfarrer, engagierte Lokalpolitiker und laienhafte Heimatforscher Heinrich Hansjakob in Hagenau Hermann den Lahmen als „leidenschaftlichen Zeitgenossen und Zeitkritiker“. Er habe sich zu den akuten Fragen der Zeit eine begründete Meinung erarbeitet, „zwar mit Hilfe gelehrter Tugenden, wie Belesenheit und Genauigkeit, aber nicht um ihretwillen, sondern angeregt von Gemeinschaften seiner engeren Heimat seine gräfische Herkunft aus der nördlichen Bodenseeregion und besonders sein Mönchskonvent auf der Insel Reichenau“. Hermann wurde ein großer Gelehrter - so Hansjakob - „wenn er über intellektuelle Kenntnisse hinaus zur moralischen Wirkung strebte und seine sinnliche Überzeugung standfest vertrat". 33

Heute wird kaum von jemandem bezweifelt, dass Hermann der Lahme „einer der größten Gelehrten des Mittelalters“ war, und dass er „das beste Geschichtsbuch des 11. Jahrhunderts verfasste“. ${ }^{34}$ Gegenwärtig wird nicht nur der Umstand besonders hervorgehoben, dass er besonders schwer „behindert“ war, sondern auch seine Leistungen - nicht nur zur Geschichtsschreibung, sondern auch zur Computistik ${ }^{35}$ - werden gewürdigt.

Hermann der Lahme, von klein auf spastisch gelähmt, ${ }^{36}$ geboren 1013 und gestorben 1054, wurde von seinen Zeitgenossen respektiert und geliebt. Schon zu Lebzeiten wurde er als Heiliger verehrt. Er gilt noch heute als ein großer Dichter und Musiker, ${ }^{37}$ und seine Lieder werden zum Teil noch heute gesungen. Außerdem beschäftigte er sich eingehend mit Musik, Astronomie, Mechanik und Mathematik. ${ }^{38}$ Sein bekanntestes Werk ist das Chronicon, eine von Christi Geburt bis Mitte 1054 reichende Weltchronik in Form von Annalen, die erste genaue Chronographie seit der Antike. Mönche, Päpste, Fürsten und Kaiser bewunderten ihn. Er wurde mit der höchsten Ehrenmedaille ausgezeichnet, die das Mittelalter zu vergeben hatte: Er wurde kurz nach seinem Tod heilig gesprochen und im Petersdom begraben. ${ }^{39}$ Sein Schüler Berthold, der ihn als „heros magnus“ bezeichnet, schreibt Folgendes über ihn:

„Seine Glieder waren auf so grausame Weise versteift, dass er sich von der Stelle, an die man ihn setzte, nicht wegbewegen, nicht einmal auf die andere Seite drehen konnte. Obwohl er auch an Mund, Zunge und Lippe gelähmt war und nur gebrochene und schwer verständliche Worte langsam hervorbringen konnte, war er seinen Schülern ein beredter und eifriger Lehrer, munter und heiter in der Rede, in der Gegenrede äußerst schlagfertig, zur Beantwortung von Fragen immer bereit. Stets glaubte dieser Mensch ohne Tadel, sich alle Fähigkeiten aneignen zu müssen, ob er nun mit seinen ebenfalls gekrümmten Fingern etwas Neues aufschrieb, ob er für sich oder mit andern etwas Geschriebenes las, oder ob er sich mit ganzer Anspannung an irgendeine nützliche oder notwendige Arbeit machte. ... Keiner

33 Borst (1988a), S. 136. Vgl. Hansjakob (1875).

34 Borst (1988a), S. 137.

$35 \mathrm{Vgl}$. Borst (2004). - Hermann übernahm von den Arabern in Spanien eine in Europa in Vergessenheit geratene altgriechische Erfindung, dank der es inm gelang die Zeit genauer zu bemessen: den Astrolab. Vgl. dazu Borst (1988b) und (1989).

$36 \mathrm{Vgl.} \mathrm{Oesch} \mathrm{(1956).}$

37 Vgl. Klaper (2003).

38 Zu den lateinischen Texten vgl. http://flaez.ch/hermannus/index.html.

39 Borst (1990), S. 25-58. 
verstand wie er, Uhren zu machen, Musikinstrumente zu bauen, mechanische Arbeiten auszuführen. Mit diesen und vielen andern Dingen, deren Aufzählung zu lange dauern würde, beschäftigte er sich ständig, soweit es überhaupt sein schwacher Körper zuließ.“^o

Wie seinen Schülern, wäre im Mittelalter niemandem in den Sinn gekommen, den schwerbehinderten Hermannus als krank zu bezeichnen, ihn für das ganze Leben in einem Asyl einzusperren oder gar der „Euthanasie“ preiszugeben. Im Gegensatz dazu zerbrach man sich Mitte des 20. Jahrhunderts den Kopf darüber, welche Krankheit er wohl gehabt habe. ${ }^{41}$

\section{Einschub: Heinrich der Löwe}

Aus dem ständigen Bestreben der NS-Funktionsträger, die pausenlose Aufmerksamkeit des Führers zu erwecken und zu erhalten, erklärt sich der wissenschaftlich kaum vorbereitete Befehl des braunschweigischen Ministerpräsidenten Dietrich Klagges zur Öffnung der Löwengruft im Braunschweiger Dom. Im Laufe der frühen Neuzeit hatte man schon mehrfach Untersuchungen vorgenommen, so dass man 1935 nicht auf eine unangetastete Anlage traf. Die „archäologische“ Ausgrabung wurde zudem unter Ausschluss der Öffentlichkeit vorgenommen, und erst nachträglich zog man einen Landesarchäologen hinzu. Der zu Tage geförderte Steinsarkophag wurde bedenkenlos Heinrich dem Löwen zugeordnet. Die zudem gefundene Leiche in jener Höhle im Holzsarg deutete man als Mathilde, seine Gattin. Die anthropologischen Befunde, die überhaupt nicht wissenschaftlich diskutiert wurden, lassen solche Interpretationen jedoch als unhaltbar erscheinen. Das Ergebnis der Gruftöffnung war ohnehin ernüchternd genug, für alle war sie eine Riesenenttäuschung: kein blonder germanischer Krieger und Riese trat zum Vorschein, sondern ein eher schmächtiges Skelett mit dunklem Haar und einer Hüftluxation, was bedeutete, dass jenes gefundene Knochengerüst einer hinkenden Person gehört haben muss. War das der heldenhafte Löwe? Dieser Frage ging man besser nicht nach. Zahlreiche NS-Größen, darunter auch Hitler, waren zur Besichtigung der sterblichen Überreste nach Braunschweig gekommen. Schaulustigen Besuchern wurden „Locken Heinrichs des Löwen“ gezeigt. Über andere Details hüllte man sich in Schweigen.

Auf Betreiben von Klagges wurde der Braunschweiger Dom bis 1940 zur nationalen Weihstätte und zum „Staatsdom“ umgestaltet. Himmler, der noch über seine eigene Reinkarnation Heinrich des Löwen nachgrübelte, mag von den Braunschweiger Befunden nicht gerade begeistert gewesen sein. Umso kraftvoller fiel der monumentale Bau einer neuen Gruftanlage Heinrichs in grauem Granit aus.

Nun galt Heinrich der Löwe nicht mehr als Vorkämpfer der Ostkolonisation. ${ }^{42}$ Jetzt wurde seine Doppelgesichtigkeit betont: Ein Verräter an Kaiser

40 Zitiert nach Berner (1985), S. 27 f.; lateinischer Text: http://flaez.ch/hermannus/vita.html.

41 Oesch (1956).

42 Um die Vorgehensweise der Nazis im Osten zu begreifen, muss man sich klarmachen, dass der Ostkampf in ihren Augen ein Kolonialkrieg war; die Slawen galten als Sklaven (nicht nur etymologisch) und als Untermenschen; ein Gedanke, weit entfernt vom Mittelalter und von Heinrich dem Löwen. - Vgl. Lindqvist (2002), S. 227. 
und Reich in der Stunde von Not und Gefahr, ein Eidbrüchiger, ein Wegbereiter der „Rassenmischung“ im Osten. Hitler selbst kritisierte Heinrich als „Kleinsiedler“, der nicht das „Format der deutschen Kaiser“ gehabt hätte. Eine SS-Division hieß „Hohenstaufen“ (und nicht „Welfen“). Der Übergriff auf die Sowjetunion im Jahr 1941 trug den Namen „Unternehmen Barbarossa“. ${ }^{43}$

\section{Rassismus, Sexualisierung und Normalität}

„Was ist der Rassismus letztendlich? Zunächst ein Mittel, um in diesen Bereich des Lebens, den die Macht in Beschlag genommen hat, eine Zäsur einzuführen: die Zäsur zwischen dem, was leben, und dem, was sterben muss. “44

Nachfolgend möchte ich die These untersuchen, dass sich Rassismus und die Diskriminierung von Menschen mit Behinderung praktisch kaum unterscheiden (s. Abb. 1).

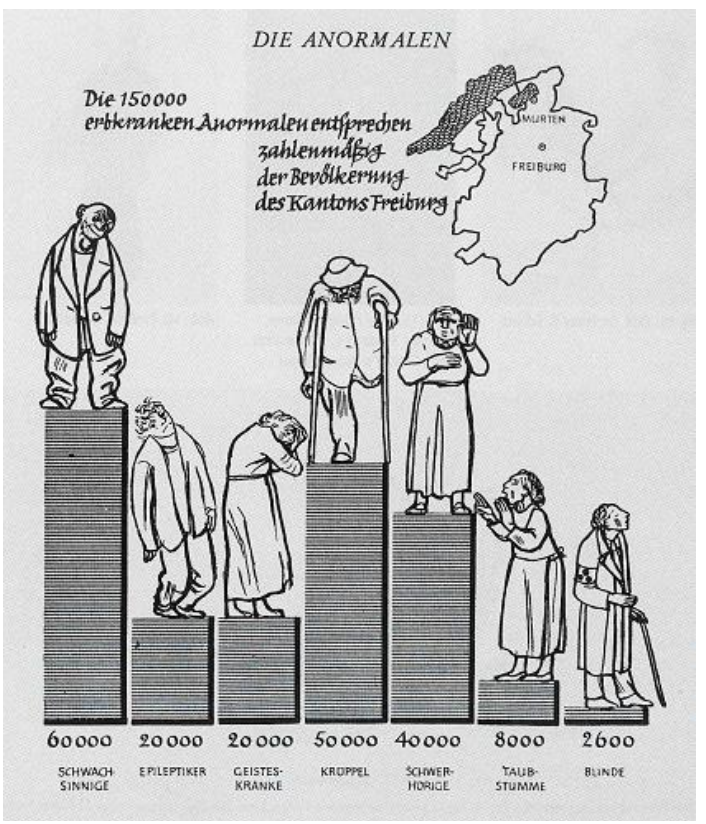

\section{Abb. 1 Die Anormalen, Grafik von Felix Hoffmann in der eugenischen Propagandaschrift von Schmid (1939)}

„Die Schweiz zählt rund
200.000 Anormale, von
denen etwa 150.000 als
erbkrank zu betrachten
sind. Zu ihnen gesellen
sich schätzungsweise $40-$
bis 60.000 Alkoholiker, die
zu einem großen Prozent-
satz gleichfalls als erblich
schwer belastet aufge-
faßt werden müssen.“

„Die Schweiz zählt rund 200.000 Anormale, von denen etwa 150.000 als erbkrank zu betrachten sind. Zu innen gesellen sich schätzungsweise 40bis 60.000 Alkoholiker, die zu einem großen Prozent satz gleichfalls als erblich faßt werden müssen."

Im Jahr 1800 kommt der 1772 in Lyon geborene „Philosoph und Menschenfreund“ Joseph-Marie Degérando in seinen Erwägungen über dieverschiedenen Methoden der Beobachtung der wilden Völker im Kapitel Über die Gebärden der Taubstummen zum Schluss, dass Taubstumme im Crunde nichts anderes seien als „zivilisierte Wilde“. ${ }^{45}$ Dass die „Wilden“ unglücklicher als die zivilisierten Europäer seien, ihre Unterle-

43 Vgl. Schneidmüller (2000), S. $297 \mathrm{f}$.

44 Foucault (2001), S. 301.

45 Degérando, in: Moravia (1973), S. 219-251. 
genheit erkannt hätten, sich mit Scham betrachten und ohne Unterlass nach europäischen, für sie unerreichbaren Idealen strebten, war für ihn selbstverständlich. ${ }^{46} \mathrm{Am}$ Schluss seiner Erwägungen vergisst er nicht, darauf hinzuweisen, dass es von wissenschaftlichem Wert wäre, einige dieser lebendigen Exemplare aus den Kolonialgebieten mit nach Hause zu nehmen (s. Abb. 2 und 3). ${ }^{47}$

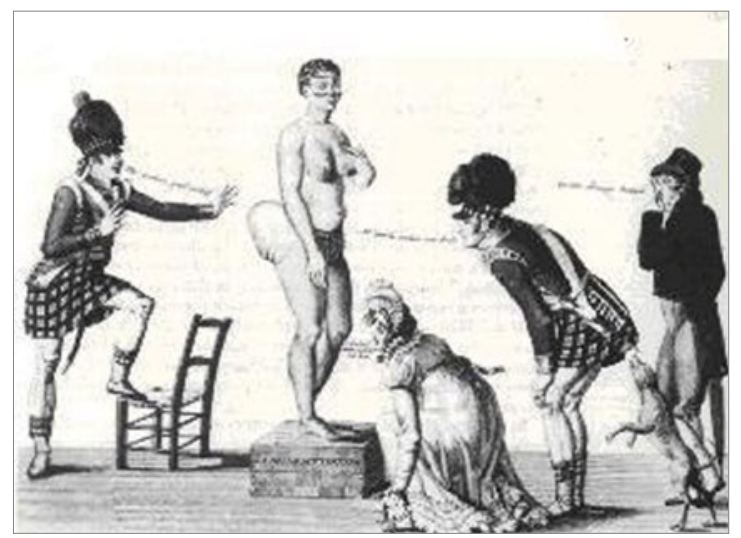

Abb. 2 Die Hottentotten in Paris

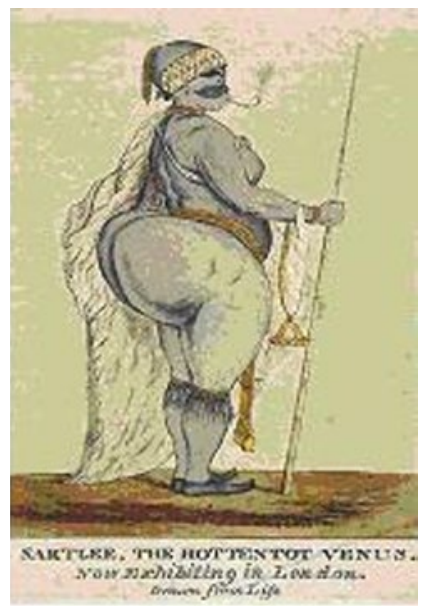

Eine der bekanntesten „wissenschaftlichen Trophäen“ war die 1815 in Paris verstorbene und als „Vénus Hottentotten“ bekannte Buschmännin Sarah Baartman, die über Jahre hinweg durch europäische Städte wegen der Größe ihres Gesäßes und ihrer Geschlechtsteile als „Ikone“ der sexualisierten schwarzen Frau einem gut zahlenden Publikum vorgeführt wurde. Nach ihrem frühen Tod wurden ihre Genitalien anatomisch präpariert, wissenschaftlich beschrie-

46 Vgl. Moravia (1973), S. 238.

47 Vgl. Moravia (1973), S. 249. 
ben und weiterhin öffentlich ausgestellt. Ihre vergrößerten Schamlippen und vor allem ihr übergroßes Gesäß wurden zum Zeichen devianter weiblicher Sexualität von Schwarzen oder von Prostituierten und schließlich als Beweis der gottgegebenen Andersartigkeit der Anderen (nicht etwa nur die Hautfarbe, sondern das radikale Gegenteil)..$^{48}$

Dieses Arrangement hatte nicht nur auf den Jahrmärkten Tradition, wo schon seit dem späten 16. Jahrhundert und bis in die 1920er Jahre Schwarze und andere sog. Monstra gezeigt wurden, ${ }^{49}$ sondern auch in Texten. ${ }^{50}$ Die 1815 in Paris ausgestellte „Hottentottin“ beschrieb der französische Naturalist und Anthropologe Julien Joseph Virey (1775-1846) in seinem Dictionnaire des sciences médicinales (1815-1841) als „exemple vivant“:

„Wir machen dazu zwei Bemerkungen. Erstens, dass man die auffallende Verlängerung der äußeren Geschlechtsteile der Afrikanerinnen mit gewissen Blumen im selben Klima vergleichen kann, zum Beispiel mit Geranien (oder pelargonium), deren äußere Blütenblätter länger sind als die inneren, vielleicht, um die Geschlechtsorgane zu bedecken und sie vor der starken Sonne Afrikas zu schützen. [...] Zweitens: Die Fettpolster beim Steißbein gleichen den Fettmassen bei den afrikanischen Schafen mit breitem Schwanz, den Höckern der Kamele und der Zebus in diesem Land."

Die Überzeugung von der grundsätzlichen Verschiedenheit und Inferiorität vor allem der "Neger“ war im 19. und 20. Jahrhundert eine selbstverständliche Annahme. ${ }^{51}$

Eine Vielzahl von Ethnien in Süd- und Nordamerika, in Afrika und in Australien wurde seit Anfang der 183oer Jahre verjagt, umgesiedelt und ausgelöscht. So war beispielsweise der vollständige Genozid an den Tasmaniern im 19. Jahrhundert ein viel zitiertes Beispiel für die angebliche „biologische“ Überlegenheit der weißen Rasse. ${ }^{52}$

Der englische Anthropologe James Cowles Prichard (1786-1848), der Erfinder des Begriffes „,moral insanity“, hielt es 1838 in seiner Vorlesung „Über die Auslöschung der Menschenrassen“ für unmöglich, „die wilden Rassen“ zu retten. Statt unnötig Kraft darauf zu verschwenden, solle die „zivilisierte Rasse“ so

48 Sarasin (2001), S. 198; Gilmann (1992), S. 119-154; vgl. auch Badou (2001).

49 Zu sog. freak shows vgl. Bischoff (1978); Tervooren (2002). Mit der Renaissance bzw. dem Spätmittelalter begann eine neue, noch nie da gewesene Wahrnehmung der Umwelt. Besonders die Entwicklung des Buchdrucks schaffte neue Möglichkeiten der Kommunikation. Die massenhafte Einführung des gedruckten Flugblattes erweiterte den Horizont der Menschen: Relativ zeitnah erfuhr man Neuigkeiten, mit denen man früher nicht konfrontiert wurde. (Vgl. Senn [1975]). Das Flugblatt ist der Vorläufer der Massenmedien, vor allem der Boulevardpresse. Zum ersten Mal in der Geschichte war - für jeden offenbar - die Anormalität geschaffen worden. Viele Flugblätter beförderten den Glauben an Wundererscheinungen: Verbrechen und Kriege, Unwetter, Überschwemmungen und andere Naturkatastrophen, Hexen- und Werwolfprozesse (mit der obligaten detaillierten Schilderung ihrer Hinrichtung) und besonders Missgeburten, sog. Monstra. - Vgl. Sonderegger (1927).

Der armlose Artist Thomas Schweicker formulierte schon 1599 den Sinn und Zweck der Freakshow: „Dieweil ich das es gott erbarm Hab weder finger hend noch arm und mich also behelfen müs Schreib ich doch dis mit meinem füss Drumb frommer Christ den lebenlang Sag Gott für diese Wohlthat danck Das du hast ein geraden leib ..." - Vgl. Bischoff (1978), S. 192.

50 Zürcher (2004).

51 Sarasin (2001), S. 199.

52 Vgl. Lindqvist (2002), S. 157-160. 
viele Informationen wie möglich über deren physische und moralische Eigenschaften sammeln. Auch der schottische Arzt und Naturwissenschaftler und Begründer des angelsächsischen Rassismus Robert Knox (1791-1862) meinte, dass die „südafrikanischen Eingeborenen“ in Kürze zu „naturkundlichen $\mathrm{Ku}$ riositäten“ würden. Zum Trost versichert er seinen Lesern:

„Einen Ausgestopften haben wir bereits in England, ein zweiter steht, wenn ich nicht irre, in Paris. [...] Mit einem Wort: sie werden schon bald von der Erdoberfläche verschwunden sein. ${ }^{“ 53}$

Alfred Russel Wallace (1823-1913) vertrat die Ansicht, eine Rasse brauche umso mehr Land, um leben zu können, je weniger entwickelt sie sei. Wo die Europäer mit ihrer größeren Energie das Land der Ureinwohner übernommen hätten, müssten die niedrigeren Rassen - um überhaupt mithalten zu können schnellstmöglichst zivilisiert werden. „Zivilisation allerdings könne nur langsam und Schritt für Schritt erworben werden, weshalb das Aussterben der niederen Rassen eben doch nur eine Frage der Zeit sei. “" ${ }^{4}$

Darwin hatte sich - als er seine These vom schicksalsbestimmten Untergang bestimmter Rassen formulierte - auf allgemein bekannte historische Ereignisse bezogen. Gelegentlich war er selbst Augenzeuge gewesen, ${ }^{55}$ beispielsweise 1833 in Argentinien: Darwin erlebte Menschenjagden auf die Indios und die Gräuel der massenhaften Ermordung mit, nachdem die argentinische Regierung kurz zuvor beschlossen hatte, die Indianer der Pampa auszurotten. Er war zwar zutiefst abgestoßen von den blutverschmierten Soldaten, die er am Colorado River mitten in ihrer grausamen Indianerjagd getroffen hatte. Aber auch er bekundete später schriftlich, dass solche Ausrottungsfeldzüge gegen mindere Rassen wohl unvermeidlich seien. In seinem Buch die „Abstammung des Menschen“von 1871 vertritt er die Ansicht, dass „in einer künftigen Zeit“, die seiner Meinung nach „nicht einmal sehr weit entfernt“ sei, „die zivilisierten Rassen der Menschheit wohl sicher die wilden Rassen auf der ganzen Erde ausgerottet und ersetzt haben“ werden. ${ }^{56}$ Robert Knox gab zur Erklärung, wieso die dunkle Rasse minderwertig sei, folgende Antwort:

„Ich kann mir das nicht anders erklären, als dass die dunklen Rassen physisch und damit auch psychisch unterlegen sind. - Was weniger auf ein geringeres Gehirnvolumen als auf eine geringere Qualität der Gehirnsubstanz zurückzuführen sei. Das Gewebe des Gehirns, so scheint mir, ist bei dunkelhäutigen Rassen im Allgemeinen dunkler, der weiße Teil faseriger; allerdings beruht diese Beobachtung leider auf extrem begrenzten Erfahrungen. “57

53 Zitiert nach Lindqvist (2002), S. 187.

54 Weiter meinte er: „Wir müssen dem mit Fassung entgegen sehen. Es ist eine Wohltat der Natur, dass die Schwachen stets von den Starken verschlungen werden. Eine dankbare Nachwelt werde die Erinnerung an die Schwarzen in Ehren halten, und eines nicht allzu fernen Tages werden weinende Damen unter Palmen sitzen, The Last Negro lesen und der Niger wird, vergleichbar dem Rhein in Europa, zu einem romantischen Mythos geworden sein." Zitiert nach Lindqvist (2002), S. $191 \mathrm{ff}$.

55 Sarasin (2001), S. 155.

56 Darwin; zitiert nach Sarasin (2003a), S. 72 f.

57 Zitiert nach Lindqvist (2002), S. $183 \mathrm{f}$. 
Wie begrenzt diese Erfahrungen waren, bestätigt er an anderer Stelle. Dort meint Knox, er habe lediglich einen einzigen Farbigen obduziert. In den Armen und Beinen dieses Körpers jedoch hätten sich „nur zwei Drittel der Nerven gefunden, die ein Weißer von derselben Größe habe.“ Damit lag für ihn auf der Hand, dass auch die Seele, der Instinkt und der Verstand der beiden Rassen in einem solchen Verhältnis zueinander stehen müssten. ${ }^{58}$

Der als Begründer der Eugenik bekannte Francis Galton (1822-1911), ein Vetter Darwins, weitete die Vorstellung, dass „tieferstehende Menschen“ in der Zukunft kein Lebensrecht mehr hätten, auf Kriterien innerhalb der „zivilisierten Rasse“ aus. Die als „minderwertig“ Eingestuften waren für ihn nicht nur die „Wilden“, sondern auch „kranke“ und „schwache“ Angehörige der „zivilisierten“ Welt. Die Zivilisation selbst sei verweichlichend und fördere die „Kranken“ und „Schwachen“. Damit aber sei die Vorherrschaft der „Zivilisierten“ durch die Zivilisation selbst in Gefahr. Es gelte also innerhalb der „zivilisierten“Welt ebenfalls die „Wilden“ auszumerzen, damit diese sowohl „zivilisiert“ als auch „gesund“ und „stark“ bleibe bzw. wie Galton es ausdrückte, sich als „fit“ erweise.

Die Auslöschung einer artfremden Rasse setzte er nicht zwangsläufig mit „Leid“ gleich: sie habe eher - so Galton - etwas mit Apathie und Lustlosigkeit zu tun. Nachdem sie mit der Zivilisation in Kontakt gekommen seien, verlören die Generationen offenbar das Interesse aneinander. Das habe zur Folge, dass sich die Zahl ihrer Nachkommen verringere: ein unglückseliger Umstand, der trotzdem kaum als Leid bezeichnet werden könne. ${ }^{60}$

Auch der Medizinprofessor Karl Reclam setzte in seinem 1870 erschienenen populärwissenschaftlichen Buch Der Leib des Menschen auf den Platz zwischen den (weißen) Menschen und den tierischen Vorfahren als Figur des biologischen Übergangs nicht wie üblich die „Neger“ oder die „Wilden“, sondern die „Blödsinnigen“, die „Kretinen“, die „Troddeln“ und die „Mikrocephalen“, die er allesamt schlicht „Affenmenschen“" nannte. ${ }^{61}$

Benjamin Kidd legte 1894 dar, wie unvergleichlich effizient die Angelsachsen bei der Auslöschung der unterentwickelten Völker vorgingen. Instinktiv würden sie in die Fremde gehen, um dort die natürlichen Ressourcen zu erschließen - mit Begleiterscheinungen, die leider Gottes unvermeidlich seien. Für die Rasse, die in diesem Kampf die Oberhand behalten wollte, werde die Ausrottung anderer Rassen zu einer harten, aber unumgänglichen Bedingung. Natürlich - so meint er - könnte man in Ausnahmefällen die Sache auch menschlicher gestalten; grundsätzlich ändern aber ließe sie sich nicht. ${ }^{62}$

Weiter behauptet Forel, es gebe „Menschen, die zwar zoologisch zur Species homo sapiens gehören, weil sie leider mit unseren Kulturmenschen noch Mischprodukte geben“ würden, „diese Wesen“ seien „aber in geistiger Beziehung so minderwertig, dass sie nur zu einer ganz niedrigen Kulturstufe fähig“ seien. „Ihr Gehirn“ sei nämlich „viel kleiner als das unsrige“. ${ }^{63}$

58 Ebd.

59 Galton (1865); Galton (1869); Galton (1883).

60 Lindqvist (2002), S. 203.

61 Sarasin (2003 b), S. 64.

62 Lindqvist (2002), S. 202.

63 Forel (1905), S. 6. 
1911 führte Eugen Bleuler die „Schizophrenie““64 - seine Wortschöpfung - mit großem Erfolg in die Psychiatrie ein. Eine ähnliche Karriere wie die „Schizophrenie“ - wenn auch weniger erfolgreich - machte die von Bleulers Schüler und Nachfolger Hans W. Maier bereits in seiner Dissertation erfundene Diagnose „moralischer Schwachsinn“ bzw. „moralische Idiotie“. ${ }^{65}$ Seiner Meinung nach war die Behandlung dieser „moralisch Defekten“ „ganz aussichtslos“. Darum empfahl er, dass „,ihre Tötung“ auch in seiner Zeit noch „das Vernünftigste [...] und für alle Teile Schonendste“ wäre: „Wenn nur der hier durchaus unangebrachte Begriff der Strafe nicht stets damit verbunden wäre und Gründe der allgemeinen Moral gebieterisch dagegen sprächen. “ ${ }^{66}$

Wenn nicht die Tötung, so sollte wenigstens die straflose Sterilisation auf keinen Fall unterlassen werden: „Die direkte Heredität“ sei nämlich „entschieden“ die wichtigste Ursache „des krankhaften Zustandes“. Darum sei es „sehr wichtig, diese Kranken an der Fortpflanzung zu hindern“. Da aber „unter unseren heutigen Verhältnissen“, weder „Gefängnis“ noch „Anstalt“ dafür „absolute Garantie bilden“ würden, „hätte die Gesellschaft nicht nur das Recht, sondern [seines] Erachtens auch die Pflicht, hier möglichst bald die zwangsweise Sterilisierung zu dekretieren. “67

Eine willkürliche Diagnose ${ }^{68}$ eines Psychiaters reichte aus, um einer schwangeren Patientin einen Abort mit der obligaten Sterilisation oder keineswegs ungefährlichen Kastration ${ }^{69}$ „anzuraten“, sie mit einem Eheverbot zu belegen und für den Rest ihres Lebens in eine Psychiatrie zu sperren, ${ }^{70}$ wo unter anderem Briefzensur, Isolation, Zwangsjacken, Insulinkur, zehntägige Schlafkur, Wickel, psychochirurgische Eingriffe, Dauer- und Deckelbad, „schwarze Spritzen“ mit unbekanntem Inhalt (die stundenlanges Erbrechen bewirkten), Hormontherapien, ${ }^{71}$ Schläge und andere Zwangsmaßnahmen ${ }^{72}$ auf sie warteten. ${ }^{73}$

Zwar sanken die eugenischen Maßnahmen (Eheverbote, Aborte, Sterilisation und Kastration) nach 1945, wurden aber dennoch, jetzt ohne öffentliche Begleitpropaganda, nun stillschweigend, zum großen Teil von den gleichen Akteuren, weitergeführt. ${ }^{74}$ So schrieb 1969 der Oberarzt der Frauenklinik in Graz:

„Heute wird die Sterilisation in diesem Lande nach mehreren schweren Geburten und körperlicher Erschöpfung, beim zweiten bzw. dritten Kaiserschnitt, bei Vielgebärenden, chronisch Kranken und Psychopathen sowie bei Frauen in schlechten sozialen Verhältnissen durchge-

64 Der Begriff „Schizophrenie“ löste die Diagnose „Dementia praecox“ ab, die von Kraepelin, dem Begründer der heute noch größtenteils gültigen psychiatrischen Terminologie. - Vgl. Kolle (1956), S. 175-186.

65 Maier (1908).

66 Maier (1908), S. 79.

67 Arnold (1992), S. 67.

68 Vgl. dazu, Rufer (1997), S. 25 f.

69 Fürst (1929), S. 53-58; vgl. Meier (2002), S. 143-160; vgl. auch Wottreng (1999).

70 Vgl. die tragische Fallgeschichte der Marta B., in: Huonker (2003), S. 200 f. - Vgl. auch die dramatische Biographie von Friedrich Glauser auf den Seiten 64 bis 78. In seinem Fall hatte sich die Amtsvormundschaft durchgesetzt. Der „Psychopath“ Glauser hatte weder geheiratet, noch Kinder gezeugt. Er starb 1938.

$71 \mathrm{Vgl}$. zum tragischen Tod von Alan Turing: Hodges (1994).

72 Vgl. Meier (2002).

73 Roth (1948).

74 Vgl. Huonker (2003), S. 149-157. 
führt [...], wobei man behördlicherseits den Standpunkt vertritt, dass die Sterilisation aus medizinischen oder eugenischen Gründen eine rein ärztliche Angelegenheit sei. ${ }^{\text {c75 }}$

Die Kontinuität der Schweizer Eugenik bestand jedoch nicht nur in der Praxis weiter, auch der eugenische Diskurs wurde nach 1945 fortgeführt.

In seinem 1945 veröffentlichten Aufsatz, in welchem er deutlich an der Theorie von unwertem Leben festhielt, meinte der bereits erwähnte Jurist Oswald Rohrer:

Im Wesentlichen würde die dritte Schicht von Behinderten „die Blödsinnigen aller Grade und Variationen von da an, wo die menschliche Intelligenz erlischt, bis hinunter zu einem rein vegetierenden Dasein“ umfassen. „In den untern Lagen dieser Schicht stellt jedes einzelne Individuum eine Last für seine Umgebung bzw. für die menschliche Gesellschaft insofern dar, als es eine große Summe von pflegerischer Kraft und Mitteln verbraucht, ohne dass dieser Aufwand einem geringsten Gegenwert entsprechen würde, und vor allem, ohne dass dieses Individuum selbst irgendeinen menschlichen Genuss von seinem Leben hätte. “76

1946 publizierte Jakob Wyrsch seine „Gerichtliche Psychiatrie“, worin er weiterhin auf eugenische Zwecke von Sterilisation und Kastration verwies. ${ }^{77}$

Der Jurist Fritz Hauser befasste sich in seiner Dissertation 1952 an der Universität Zürich mit der „Frage der Euthanasie im schweizerischen Strafrecht“. Seine Schlussfolgerungen wichen nicht wesentlich von jenen der beiden NaziProfessoren Binding und Hoche $(1920)^{78} \mathrm{ab}^{79}$

Die Schweizer Ärztin Paulette Brupbacher meinte in ihrer 1953 erschienenen Schrift über die Legalisierung des Schwangerschaftsabbruchs, ohne eugenische Indikation würde „der Gemeinschaft die Erhaltung körperlich oder geistig

75 Heiss (1969), S. 25-79.

76 Rohrer (1945), S. 27.

77 Wyrsch (1946), S. 259-266. - Unter „Krüppelhaftigkeit“ - im Kapital „Abnorme Zustände“ (S. 45-81) - verstand Wyrsch: „Der Charakter des Krüppels hat sein besonderes Gepräge. Das Gefühl der körperlichen Benachteiligung und die Erfahrung der Abhängigkeit und Unfreiheit führen zu Misstrauen, zu Unzufriedenheit, zu Verbitterung und dem Bewusstsein überflüssig zu sein, und auf diesem Boden wachsen Beeinträchtigungsideen und, als Kompensation der Mängel, auch Boshaftigkeit und Widerspenstigkeit. Ist der Behinderte ehrgeizig und willenskräftig, äußert sich das Genannte in Trotzhandlungen, in sexueller Haltlosigkeit und auch in antisozialer Lebensführung. Sexualdelinquenten, und zwar sowohl Täter wie Opfer, sind nicht selten solche im Leben Benachteiligte. Ein anderes ungeheures dichterisches Beispiel einer solchen Entwicklung ist Shakespeares Richard III. Sinnesschwache, Taubstumme, Verkrüppelte oder mit chronischen unheilbaren Krankheiten Behaftete brauchen deshalb besonders verständnisvolle Fürsorge, und man darf ihnen nicht jede Unart und jede reaktive Verstimmung gleich nachtragen, will man Schlimmeres vermeiden." (ebd. S. 250 f.)

78 In ihrer 1920 veröffentlichten Schrift Die Freigabe der Vernichtung unwerten Lebens, ihr Maß und ihre Form vertraten Binding und Hoche Postulate, die die Straffreiheit bei Sterbehilfe, andererseits die Vernichtung unwerten Lebens forderten; vgl. Müller (2001), S. 203-220.

79 „Obwohl ich grundsätzlich gegen die vollständige Freigabe der Vernichtung solcher Leben bin, möchte ich doch in gewissen krassen und schweren Fällen eine Tötung im Sinne einer Erlösung für die betreffenden Geschöpfe und im Interesse der Eltern bzw. der Angehörigen befürworten. Es lässt sich nicht bestreiten, dass es Fälle gibt, in denen der geistige und körperliche Zustand nicht mehr als menschenwürdig und menschenähnlich bezeichnet werden kann. Ich denke hier z. B. an Kinder von Geschlechtskranken und Alkoholikern, deren Anblick man oft kaum ertragen kann und die gar nicht lange lebensfähig sind. Ferner gehören auch die schweren Formen des angeborenen Schwachsinnes hierher, bei denen der Betreffende nicht einmal mehr auf der Stufe der höher entwickelten Tiere steht. Hier sollte es irgendeine Möglichkeit geben, solche Menschen zu erlösen, ohne deswegen strafrechtlich zur Verantwortung gezogen zu werden." - Vgl. Hauser (1952), S. 48. 
unbrauchbarer, lebensuntauglicher Elemente zugemutet, die, nirgends eingereiht, ihr nutz- und freudloses Dasein in Heimen verdämmern. “80

Unangefochten und besonders aktiv blieb der zum Professor an der Universität Zürich arrivierte Präsident des Zürcher Kantonsrats (1955-1959) und ehemalige Mitherausgeber des Nazistandardwerks Handbuch der Erbbiologie des Menschen, ${ }^{81}$ Ernst Hanhart. Er erforschte weiterhin die „Erbbiologie von Gehörlosen“" ${ }^{82}$ und von „Mongoloiden" ${ }^{* 83}$ und notierte noch in den $1970 e r$ Jahren stolz die Erfolge von „Eugenischen Beratungen““. ${ }^{84}$ Seine Studenten durchsuchten die Bevölkerung von Bergdörfern nach verdächtigen „Sippen““. 85

\section{Die Sexuelle Anomalie}

1946 teilte der Berner Professor für Psychiatrie Jakob Wyrsch „die sexuellen Verirrungen (Perversionen) und die Sexualneurose“ein in: „Exhibitionismus“, „Sadismus und die Masochismus“, „Pädophilie“, „Sodomie (Bestialität)“, „Fetischismus“, „Nekrophilie“, „Transvestismus“, „Homosexualität (konträre Geschlechtsempfindung)“ - von denen die meisten oder wenigstens viele „an sich schon psychopathisch veranlagt“ seien - und „Hermaphroditismus“. „Ueberall“ handle es sich nämlich nur „um Ersatzbefriedigung für das normale Sexualziel, die Vereinigung mit dem Geschlechtspartner, die aus [...] neurotischen Gründen nicht erreicht wird“. Diese „Anomalien“ seien durch strikte Enthaltung - oder noch besser: durch Psychotherapie bzw. Einweisung in eine Psychiatrie - zu heilen. Auf jeden Fall rät Wyrsch von einer (heterosexuellen) Zwangsheirat dringend ab, denn sie seien „dafür nicht geschaffen“. Da auch die Erwartungen, die man noch zwanzig Jahre zuvor an die „Implantation eines fremden Hodens bei Homosexuellen“"geknüpft hatte, sich „nicht erfüllt“ hätten, empfiehlt auch er diese Methode nicht. ${ }^{86}$

Zur Zeit der Industrialisierung Europas wurde die abenteuerliche Verbindungslinie zwischen dem zweiten Hauptsatz der Thermodynamik ${ }^{87}$ und dem, was man als ersten Hauptsatz der Sexualität im 19. Jahrhundert bezeichnen kann - dem absoluten Verbot der Masturbation -, gezogen. Man befürchtete, dass durch sie vor allem der männliche Körper so viel Energie verlieren würde, dass der physische und geistige Zerfall ${ }^{88}$ schon in jungen Jahren unausweichlich sei. ${ }^{89}$

Diese bizarre Imagination, die schon am Ausgang des 17. Jahrhunderts formuliert worden war, als „Sünde Onans“90 verdammt wurde und empfindsame

\footnotetext{
80 Brupbacher (1953), S. 232.

81 Just (1939).

82 Huonker (2003), S. 156.

83 Hanhart (1960).

84 Hanhart (1972).

85 Huonker (2003), S. 156.

86 Wyrsch (1946), S. 208-218.

87 Der zweite Hauptsatz der Thermodynamik beschreibt die konstante Abnahme der produktiven Energie in einem geschlossenen System (Entropie).

88 Im Allgemeinen werden Auszehrung, Epilepsie und „Blödsinn“ genannt.

89 Sarasin (2003 a), S. 94.

90 Stolberg (2003).
} 
Gemüter zuweilen tatsächlich in Panik versetzte, fand ihren Höhepunkt in der Dissertation von Samuel Auguste Tissot (1728-1797) aus Lausanne über die Gefahr des „Onanisme“: eine sexuelle Energielehre des männlichen Körpers, die von 1760 bis 1905 immer wieder neu aufgelegt und in der gesamten hygienischen, psychiatrischen und medizinischen Literatur der Zeit zitiert wurde.

„Unser Körper“, so beginnt Tissot seine Schrift, „verliert beständig. Könnten wir den mannichfaltigen Verlust, den wir daran leiden, nicht ersetzen, so würden wir gar bald in eine tödtliche Schwachheit verfallen“. Der alltägliche Verlust lasse sich mit „Speise und Trank“ ersetzen, die für die „Kräfte des Körpers“ wertvolle „Saamenfeuchtigkeit“ jedoch nicht: Sie bildet einen „Vorrath“, mit dem man haushälterisch umgehen soll. Auch die körperlichen Folgen derjenigen, „die sich in einer natürlichen Beywohnung erschöpfen“, seien „schrecklich“ - existenzgefährdend allerdings seien die unkontrollierten Verluste durch Selbstbefleckung. ${ }^{91}$

Zur Verhinderung der ,Schizophrenie“ empfiehlt Eugen Bleuler: „Onanie, Liebesgram, Überanstrengung, Schreck [...] vermeiden“ könne er mit gutem Gewissen anraten, „weil es sicher auch sonst gut ist, sich vor diesen Dingen zu hüten“. Allerdings räumt Bleuler ein, „dass jemals durch solche Vorsicht eine Schizophrenie am Ausbrechen verhindert wurde“, sei nicht zu belegen. Allerdings werde man ,ja unter Umständen“ kaum jemals „ganz auf die diätetische Bekämpfung der Onanie verzichten und daneben kühles und nicht zu weiches Lager verordnen." 92

Nach Eugen Bleuler ist die Onanie nicht nur ein Symptom der ,Schizophrenie‘, sondern auch die Hauptursache dafür: Zur Behandlung empfiehlt er die Sterilisation (,Bougieren' und ,Tuschieren'). ${ }^{93}$

Für die Psychiatrie des beginnenden 20. Jahrhunderts galt nicht nur die ,Onanie‘ als Krankheit und klares Zeichen für die Zugehörigkeit zur Gruppe der ,Anormalen', ${ }^{94}$ auch andere sexuelle ,Perversionen' suchte man mit Sanktionen zu belegen; ${ }^{95}$ besonders gilt es - da ja bekanntlich alle Abartigkeiten als vererbt angenommen wurden - die Fortpflanzung zu verhindern.

„Ist ein Masochist mit einer Sadistin verheiratet, so ist ja den beiderseitigen Wünschen Rechnung getragen und in der Regel eine Einmischung in die Ehe völlig unnötig. Bedenklicher schon ist die Fortpflanzung solcher Paare, da wir unter ihren Nachkommen eben häufig konstitutionell Abnorme finden. So hatte z. B. nach einer eigenen Beobachtung ein masochis-

91 Stolberg (2003), S. 94-95.

92 Bleuler (1911), S. 380-382.

93 Ebd.

94 „[...] kommt es doch hier in erster Linie darauf an, unsere grundsätzliche Einstellung gegen abnorme Sexualität als einer krankhaften Erscheinung zu begründen, als alle Möglichkeiten einer Beschreibung zu erschöpfen. [...] Bei Geisteskranken und Schwachsinnigen finden wir die Nekrophilie.. [...] die Zoophilie ist [...] nichts anderes als ein sexueller Notakt Schwachsinniger". - Vgl. Forel (1931), S. 280.

95 „Wir müssen [...] daran festhalten, dass die homosexuelle Liebe krankhaft ist und dass [...] alle Urninge auch sonst mehr oder minder schwere Psychopathen, besonders Hysteriker, sind." (Forel [1931], S. 274). Weiter meint Forel: „Leider sind Dauererfolge verhältnismäßig selten“, darum sind sie bei Exhibitionisten „am ehesten noch durch Kastration zu erwarten. [...] Schwere Exhibitionisten sollten interniert werden, wenn Behandlungsversuche nicht zum Erfolg geführt haben“. Dasselbe gilt auch „wenn der Mann Transvestit ist, usw. Da es sich nach allem, was ich zu sehen Gelegenheit hatte, stets um konstitutionell abgeartete Menschen handelt, wird man jedoch Fortpflanzung widerraten müssen." (Forel [1931], S. 263-265). 
tischer Mann, der erst dann zum Beischlaf fähig war, wenn er mit Sporen blutig geschlagen wurde, mit seiner sadistischen Frau zwei Söhne gezeugt. Der eine ist ein minderwertiger Homosexueller, der andere geisteskrank (Schizophrenie) in einer Anstalt gestorben. ${ }^{966}$

Wie bei der sexuellen Anomalie schlechthin, sah er auch die Ursachen der Homosexualität im Alkoholmissbrauch. ${ }^{97}$

Forel notiert eine tragische Geschichte über zwei weibliche ,Urninge“: „Ganz allmählich, durch geschickt herbeigeführte Steigerung“, so beginnt Forel, „bringt es oft der weibliche Urning dazu, bei seinem Opfer (sic!) Wollustempfindungen durch Küssen der Brustwarzen und durch Reibung der Klitoris hervorzurufen“. Erstaunt stellt Forel fest, dass „die Geliebte sich in der Regel [...] der Abnormität der ganzen Sache nicht recht bewusst wird und sehr leicht schwärmerisch verliebt bleibt“.

Weiter erzählt Forel einen angeblich selbst beobachteten und für ihn gänzlich unverständlichen Fall von zwei „schwärmerisch verliebten Urnings“: „Ein normales Mädchen wurde [...] von einem als Mann verkleideten weiblichen Urning getäuscht und in ein Liebesverhältnis verwickelt, das in einer formellen Verlobung seinen vorläufigen Abschluss fand“. Später wurde „die Betrügerin ertappt, verhaftet und dann zur Beobachtung in die Irrenanstalt versetzt“. Gänzlich fassungslos notiert Forel, dass „das normale Mädchen“ auch nach der „Entlarvung“ verliebt war; sie „besuchte ihren ,Liebling“, der, nun weiblich gekleidet, ihr um den Hals fiel und sie in einer erotisch wollüstigen Weise, die kaum zu beschreiben ist, vor allen Leuten abküsste“. ${ }^{98}$

Die „Betrügerin“ wurde also in die Psychiatrie gesteckt, sehr wahrscheinlich für den Rest ihres Lebens. Forel begründet solche Schritte folgendermaßen: Homosexualität sei zwar ebenso wenig wie Geisteskrankheit ein Anlass zur Verfolgung. Die Gesellschaft müsse aber wie vor den ,Kranken“, so auch vor den ,Urnings“ geschützt werden. Beide hätten „Anspruch auf unsere Hilfe und Verständnis, die Gesellschaft aber hat Anspruch auf Schutz vor ihnen, soweit ihr Schaden droht." ${ }^{99}$

Von allen seit Beginn der Neuzeit beschriebenen Monstra nahm eines davon eine herausragende Stellung ein: der Hermaphrodit. Im Jahr 1599 stößt man auf den Fall eines Hermaphroditen, der offensichtlich nur aus dem Grund, dass er ein solcher war, verurteilt wurde. Nach ihrer Visite einigten sich die Fachmänner - mehr Theologen als Mediziner - darauf, dass dieses Individuum in der Tat beide Geschlechter besaß. Diese könne er nur haben, weil er Beziehungen zum Teufel unterhalten hätte; und diese Beziehungen zum Satan hätten seinem ursprünglichen Geschlecht noch ein weiteres hinzugefügt. Danach gefragt, gestand der Hermaphrodit - sehr wahrscheinlich, wie damals üblich, unter Folter -, tatsächlich Beziehungen mit dem Teufel unterhalten zu haben. Daraufhin wurde er bei lebendigem Leib verbrannt. ${ }^{100}$ Das Monstrum nahm in der Zeit der Hexenverfolgung in derTat eine deutliche Beziehung

\footnotetext{
96 Forel (1931), S. $258 \mathrm{f}$.

97 Forel (1931), S. 230-283; z. B. S. 278, 264.

98 Forel (1931), S. 278-279.

99 Forel (1931), S. 280.

100 Foucault (2003), S. 93.
} 
zur Hexerei an. ${ }^{101}$ Diesem Fall sollten im Verlauf der frühen Neuzeit zahlreiche andere folgen. ${ }^{102}$

Einen Zusammenhang zwischen „Anomalie“ und Delinquenz wollte Cesare Lombroso (1835-1909) im Jahr 1890 beobachtet haben:

„Der Idiot lässt sich von Zornausbrüchen zu Körperverletzungen und Todtschlag, von geschlechtlicher Erregung zu Nothzucht, von der Lust am Anblick des Feuers zu Brandstiftung hinreißen. [...] Der Demente hält, aus Vergesslichkeit, sein Wort nicht und schwört falsch; er begeht auch Gewaltthaten, Morde u. s. w. im Zustande gereizter Hirnthätigkeit. “103

„Alle Sexualstraftäter sind geisteskrank“ lautet ein gängiges Vorurteil. Bleuler kehrte diese These um und zog den fatalen Schluss, dass alle „Geisteskranke“, alle „Anormalen“ auch Sexualverbrecher seien. Zur Lösung dieses Problems schlug er die Vernichtung aller „Anormalen“ vor. Mit der Hinrichtung würde nämlich, so Bleuler, die Gesellschaft einerseits von der Sorge über die Delinquenten befreit und zudem verändere sie „die Zeugung einer ähnlich gearteten Nachkommenschaft“ ${ }^{104} 1942$ stellte Edmund Mezger im Abschnitt über „Schwachsinnigen-Verbrecher“ fest: „Schon früh ist den Beobachtern die hohe Beteiligungsziffer der Schwachsinnigen an der Kriminalität nicht entgangen."105

\section{Epilog}

Das Ziel dieses Aufsatzes war die Beantwortung der Frage nach der Geschichte und weiter nach dem Sinn und Zweck von „Heimen“ bzw. Asylen für „Behinderte“. Die Bezeichnung Behinderte entstand erst im Verlauf des Eugenikdiskurses und war nichts anderes als eine Weiterentwicklung des Rassismus. Sie wurde - in Deutschland - von den Nazis eingeführt; es ist kein Zufall, dass in der Schweiz von einer Wohltätigkeitsorganisation, der „Vereinigung für Anormale“, ${ }^{106}$ die neue Bezeichnung übernommen wurde, welche sich mit der Einführung auch selber einen neuen Namen gab: Und zwar geschah dies zu einer Zeit, als die Asylidee schon entstanden war - aber sich noch nicht endgültig durchgesetzt hatte. Wie sich die genannte Vereinigung bezeichnete, so wurden alle Menschen mit einer Beeinträchtigung genannt: anormal, abnormal, anomal ... ${ }^{107}$ Der etymologische Hintergrund des Wortes „normal“ ist das altrömische Wort norma, ein Ausdruck aus der Architektensprache. Normal war, was sich weder nach links noch nach rechts neigte, sich in der

101 Ewinkel (1995), S. 190-197.

102 Ewinkel (1995), S. 160 f. Zur Geschichte der Hermaphroditen, die bereits in der Antike getötet wurden: vgl. Wacke (1989).

103 Lombroso (1890), S. 254.

104 Bleuler (1905), S. $74 \mathrm{f}$.

105 Mezger (1942), S. 46.

106 Schawalder (1999).

107 „Abnormal“ wurde meist pejorativ gebraucht. An die Stelle von „anormal“ oder „abnormal“ trat ab und zu die neutrale Bezeichnung „anomal“. - Vgl. Bischoff (1978), S. 192. - Ein beliebter Ausdruck zur Bezeichnung von einem „schwer (sowohl körperlich wie geistig) Abnormalen“ war auch „Idiot“. 
richtigen Mitte hielt. ${ }^{108}$ Unter der imaginären Gruppe der Anormalen verstand man alles, was von der eingebildeten Norm, was vom virtuellen Durchschnitt abwich: Dazu zählte man neben den sexuell Abnormen, den moralisch Defekten, den Blinden, den Taubstummen, den Schwerhörigen, den Epileptikern, den Geistesschwachen auch die Krüppelhaften, die Invaliden, die Schwererziehbaren und die Psychopathen. Von der „Vereinigung für Anormale“ und ihrer Nachfolgeorganisation wurden ausdrücklich die „Geisteskranken“ ausgeschlossen: Als geisteskrank konnte aber fast jeder Anormale definiert werden (mit Ausnahme von Invaliden und Überlebenden von Unfällen). So galten im späten 19. und bis weit ins 20. Jahrhundert Monstra (Geburtsbehinderte) primär als geisteskrank und erst seit kurzem als Menschen mit einer angeborenen Andersartigkeit. Dasselbe galt auch für die Sinnesbehinderten; sie galten als geisteskrank, aber nicht, weil ihr „Geist“ krank gewesen wäre, sondern weil sie von klein auf als solche stigmatisiert und behandelt wurden; nicht weil sie dumm gewesen wären, sondern weil ihnen der Zugang zu den Bildungsstätten verunmöglicht wurde. Ähnliches lässt sich auch bei den psychisch Behinderten sagen: Wer als „Psychopath“ gekennzeichnet war, galt in erster Linie als geistesgestört und als „neuropathisch“; die Psychiater, oder korrekter: viele davon, versuchten primär, dessen vermutete Hirnkrankheit $\mathrm{zu}$ heilen.

Was bei der Betrachtung des Eugenikdiskurses auffällt - besonders bei dessen Unterkapitel Asylierung - ist die omnipräsente Angst vor massenhafter Fortpflanzung und folglich vor einem Überhandnehmen von „Anormalen“: Als Lösung dazu schlug man eine Sexualkontrolle, die Asylierung, vor.

In Übereinstimmung mit den meisten Autoren der disability history möchte auch ich festhalten, dass der Eugenikdiskurs keineswegs mit dem Nürnberger Prozess zu Ende ging und gegenwärtig einen Spitzenplatz in den Medien einnimmt (vgl. z. B. die Ausführungen von Peter Singer oder die Debatten um Ashley, Terry Schiavo oder den „Fall Perruche“"109). Als Einschübe habe ich deshalb zwei Beispiele aus einem gänzlich anderen, von der Eugenik und dem Sozialdarwinismus weit entfernten Zeitraum angeführt: das Exempel von Hermannus Contractus und das von Heinrich dem Löwen. Die heute noch von den meisten Nichtmediävisten abwertend belächelte mittelalterliche Lebens- und Todeseinstellung $^{110}$ erzeugte so etwas wie Chancengleichheit.

108 Müller (2001), S. 22.

$109 \mathrm{Vgl}$. Clemens Pornschlegel, in: Stingelin (2003), S. 206-227.

110 Man vergleiche die mittelalterlichen Todeseinstellungen - Die Erinnerung an den Tod war allgegenwärtig (vgl. Ohler [1990]) - mit der des späten 20. Jahrhunderts: Erfolgreich behandeln, aber armselig sterben (vgl. Kürten/ Dörner [1999]). 


\section{Literatur}

Antonovsky (1997): Aaron Antonovsky, Salutogenese. Zur Entmystifizierung der Gesundheit, Tübingen 1997

Anz (1989): Thomas Anz, Gesund oder krank? Medizin, Moral und Ästhetik in der deutschen Gegenwartsliteratur, Stuttgart 1989

Arnold (1992): Christian Arnold, Der Psychiater Hans Wolfgang Maier (1882-1945), Zürich 1992

Badou (2001): Gérard Badou, Die schwarze Venus. Das kurze und tragische Leben einer Afrikanerin, die in London und Paris Furore machte, München, Zürich 2001

Beard (1881): George M. Beard, American Nervousness, its causes and consequences. A supplement to Nervous Exhaustion, New York 1881

Berner (1985): Felix Berner, Baden-Württembergische Portraits, Stuttgart 1985

Binswanger (1905): Otto Binswanger, Über den moralischen Schwachsinn. Sammlung von Abhandlungen aus dem Gebiete der Pädagogik, Psychologie und Physiologie, 0.0.1905

Bischoff (1978): Ulrich Bischoff, Freaks, Abnormitäten, Schaustellerei, in: Jörn Merkert (Hrsg.), Zirkus - Circus Cirque, Frankfurt a. M. 1978

Bleuler (1911): Eugen Bleuler, Dementia praecox oder Gruppe der Schizophrenien, Wien 1911 (Neudruck: Tübingen 1988)

Bleuler (1905): Eugen Bleuler, Der geborene Verbrecher, München 1905

Böckelmann (1989): Franz Böckelmann, Die Gelben, die Schwarzen, die Weißen, Frankfurt a. M. 1989

Borst (1988a): Arno Borst, Barbaren, Ketzer und Artisten, München 1988

Borst (1988 b): Arno Borst, Wie kam die arabische Sternkunde ins Kloster Reichenau, Konstanz 1988

Borst (1989): Arno Borst, Astrolab und Klosterreform an der Jahrtausendwende, Heidelberg 1989

Borst (1990): Arno Borst, Ein exemplarischer Tod, in: Arno Borst (Hrsg.), Tod im Mittelalter, München 1990

Borst (2004): Arno Borst, Computus: Zeit und Zahl in der Geschichte Europas, Berlin 2004

Breidenstein (1996): Georg Breidenstein, Geschlechtsunterschied und Sexualtrieb im Diskurs der Kastration

Anfang des 20. Jahrhunderts, in: Christiane Eifert et al. (Hrsg.), Was sind Frauen? Was sind Männer?, Frankfurt a. M. 1996

Briese (2003): Olaf Briese, Angst in den Zeiten der Cholera, Berlin 2003

Brüne/Payk (2004): Martin Brüne, Theo R. Payk (Hrsg.), Sozialdarwinismus, Genetik und Euthanasie. Menschenbilder in der Psychiatrie, Stuttgart 2004

Brupbacher (1953): Paulette Brupbacher, Meine Patientinnen, Zürich 1953

Bumke (1997): Oswald Bumke, Lehrbuch der Geisteskrankheiten, München 1936

Darnton (1997): Robert Darnton, Washingtons falsche Zähne oder noch einmal: Was ist Aufklärung?, München 1997

Didi-Hubermann (1997): Georges Didi-Hubermann, Die Erfindung der Hysterie. Die photographische Klinik von Jean-Martin Charcot, Paderborn 1997

Droz (2003): Florence Droz, Praxis der Sterilisation und Kastration am Burghölzli zwischen 1937 und 1944. 17 Fallbeispiele, Zürich 2003

Erb (1893): Wilhelm Heinrich Erb, Über die wachsende Nervosität unserer Zeit, Heidelberg 1893

Ernst (1986): Cécile Ernst, Vererbung in der Psychiatrie. Erbanlage und Umwelt, Bern 1986

Ewinkel (1995): Irene Ewinkel, De monstris, Tübingen 1995

Fandrey (1990): Walter Fandrey, Krüppel, Idioten, Irre, Stuttgart 1990

Forel (1905): August Forel, Die sexuelle Frage. Eine naturwissenschaftliche, psychologische, hygienische und soziologische Studie für Gebildete, München 1905

Forel (1935): August Forel, Rückblick auf mein Leben, Zürich 1935

Foucault (2001): Michel Foucault, In Verteidigung der Gesellschaft, Frankfurt a. M. 2001

Foucault (2003): Michel Foucault, Die Anormalen. Vorlesungen am Collège de France (1974-1975), Frankfurt a. M. 2003

Frehe (2004): Horst Frehe, Historische Entwicklung der Diskriminierung, Bremen 2004 
Fuchs (2001): Petra Fuchs, „Körperbehinderte“ zwischen Selbstaufgabe und Emanzipation. Selbsthilfe - Integration - Aussonderung, Neuwied 2001

Fürst (1929): Walter Fürst, Untersuchungen über die Dosierung harter Röntgenstrahlung aus Fernfeldern bei der Behandlung des Collumcarcinoms, Habil. Med., Zürich 1929, Sonderdruck aus: Strahlentherapie. Mitteilungen aus der Behandlung mit Röntgenstrahlen, Licht und radioaktiven Substanzen, Doppelband 33/34, Berlin 1929

Galton (1865): Francis Galton, Hereditary Talent and Character, London 1865

Galton (1869): Francis Galton, Hereditary Genius, London 1869

Galton (1883): Francis Galton, Inquiries into Human Faculty and Its Development, London 1883

Gilmann (1992): Sander L. Gilmann, Hottentottin und Prostituierte. Zu einer Ikonographie der sexualisierten Frau, in: Sander L. Gilmann (Hrsg.), Rasse, Sexualität und Seuche. Stereotypen aus der Innenwelt der westlichen Kultur, Reinbek 1992

Goffman (1972): Erving Goffman, Asyle. Über die soziale Situation psychiatrischer Patienten und anderer Insassen, Frankfurt a. M. 1972

Goltz (1999): Dietlinde Goltz, Die Cholera im 19. Jahrhundert, in: Dietlinde Goltz, Albrecht Hirschmüller (Hrsg.), Niemals müßig. Symposion 19.12.1998 in Tübingen, Stuttgart 1999

Griesinger (1845): Wilhelm Griesinger, Pathologie und Therapie der psychischen Krankheiten, Stuttgart 1845

Gustav (1938): Ernst-Albert Gustav: Der Stand der Eugenikdebatte in der Schweiz 1938. Das Werk „Verhütung erbkranken Nachwuchses", Basel 1938, Med. Diss., Bern 1990

Haenel (1982): Thomas Haenel, Zur Geschichte der Psychiatrie. Gedanken zur allgemeinen und Basler Psychiatriegeschichte, Basel, Boston, Stuttgart 1982

Hagner (1995): Michael Hagner (Hrsg.): Der falsche Körper. Beiträge zu einer Geschichte der Monstrositäten, Göttingen 1995

Hanhart (1960): Ernst Hanhart, 800 Fälle von Mongoloidismus in konstitutioneller Betrachtung, Zürich 1960

Hanhart (1972): Ernst Hanhart, Nachprüfung des Erfolges von 30 eugenischen Beratungen bei Vetternehen, Mainz 1972

Hansjakob (1875): Heinrich Hansjakob, Hermann der Lahme von Reichenau Sein Leben und seine Wissenschaft, Mainz 1875

Hasler (2001): Eveline Hasler, Die Vogelmacherin, München 2001

Hauser (1952): Fritz Hauser, Die Frage der Euthanasie im schweizerischen Strafrecht, Zürich 1952

Haustein (1990): Jörg Haustein, Martin Luthers Stellung zum Zauber- und Hexenwesen, Köln 1990

Hegar (1831): Johann August Hegar, Vademecum für die Behandlung der asiatischen Cholera, Darmstadt 1831

Heiss (1969): Herbert Heiss, Die Sterilisation der Frau, Stuttgart 1969

Herkommer (1947): Agnes Herkommer, Hermann der Lahme. Hermannus Contractus, 1947

Hermle (2004): Leo Hermle, Das Degenerationsparadigma in den psychiatrischen Forschungen, in: Martin Brüne, Theo R. Payk (Hrsg.), Sozialdarwinismus, Genetik und Euthanasie, Bochum 2004

Hodges (1994): Andrew Hodges, Alan Turing. The Enigma, Berlin 1994

Howard-Jones (1972): Norman Howard-Jones, Cholera Therapy in the Nineteenth Century, Oxford 1972

Huerkamp (1985): Claudia Huerkamp, Der Aufstieg der Ärzte im 19. Jahrhundert. Vom gelehrten Stand zum professionellen Experten. Das Beispiel Preußens, Göttingen 1985

Huizinga (1975): Johan Huizinga, Herbst des Mittelalters. Studien über Lebens- und Geistesformen des 14. und 15. Jahrhunderts in Frankreich und in den Niederlanden, Neudruck: Stuttgart 1975

Huonker (2002): Thomas Huonker, Anstaltseinweisungen, Kindswegnahmen, Eheverbote, Sterilisationen, Kastrationen. Fürsorge, Zwangsmassnahmen, „Eugenik“ und Psychiatrie in Zürich zwischen 1890 und 1970, Zürich 2002

Huonker (2003): Thomas Huonker, Diagnose: „moralisch defekt“. Kastration, Sterilisation und Rassenhygiene im Dienst der Schweizer Sozialpolitik und Psychiatrie 1890-1970, Zürich 2003

Jost (1992): Hans Ulrich Jost, Die reaktionäre Avantgarde. Die Geburt der neuen Rechten in der Schweiz um 1900, Zürich 1992

Just (1939): Günther Just (Hrsg.), Handbuch der Erbbiologie des Menschen, Berlin 1939 
Kaba (2006): Marianna Kaba, Quelle place pour une perspective genre dans la "disability history“, Traverse 3 (2006), Zürich 2006, S. 47-60

Kaiser (1927): Klara Kaiser, Der Anormale im Schweizer Recht, Glarus 1927

Keller (2003): Christoph Keller, Wählt einen gesunden Ehegatten. Die lange Geschichte eines eugenischen Merkblatts, in: Christoph Keller (Hrsg.), Building Bodies, Zürich 2003

Klaper (2003): Michael Klaper, Die Musikgeschichte der Abtei Reichenau im 10. und 11. Jahrhundert. Ein Versuch, Stuttgart 2003

Klee (1989): Ernst Klee, „Euthanasie“ im NS-Staat. Die „Vernichtung lebensunwerten Lebens“, Frankfurt a. M. 1989

Koch (1891-1893): Julius Ludwig August Koch, Die psychopathischen Minderwertigkeiten, 3 Teile, Ravensberg 1891-1893

Kölle (1898): Karl Kölle, Die Fürsorge für Blödsinnige im Kanton Zürich. Vortrag, 28.11.1897 in Unterstrass vor der gemeinnützigen Gesellschaft des Bezirkes Zürich (Separatabdruck aus der Neuen Zürcher Zeitung), Zürich 1898

Kolle (1956): Kurt Kolle, Große Nervenärzte, Stuttgart 1956

Kraepelin (1918): Emil Kraepelin, Ziele und Wege der psychiatrischen Forschung, Berlin 1918

Krafft-Ebing (1884): Richard von Krafft-Ebing, Der Conträrsexuale vor dem Strafrichter, Leipzig, Wien 1884

Krafft-Ebing (1886): Richard von Krafft-Ebing, Psychopathia sexualis, Stuttgart 1886

Krafft-Ebing (1889/90): Richard von Krafft-Ebing, Angeborene konträre Sexualempfindung. Erfolgreiche hypnotische Absuggerierung homosexueller Empfindungen, Internationales Zentralblatt für Physiologie und Pathologie der Harn- und Sexualorgane 1 (1889/90), S. 7-11

Krafft-Ebing (1895): Richard von Krafft-Ebing, Nervosität und neurasthenische Zustände, Wien 1895

Kürten/Dörner (1999): Claudio Kürten, Klaus Dörner (Hrsg.): Erfolgreich behandeln - armselig sterben. Macht und Ohnmacht im Krankenhaus und Heim, Gütersloh 1999

Lindqvist (2002): Sven Lindqvist, Durch das Herz der Finsternis. Ein Afrikareisender auf den Spuren des europäischen Völkermordes, Zürich 2002

Lombroso (1890): Cesare Lombroso, Der Verbrecher (Homo Delinquens) in anthropologischer, ärztlicher und juristischer Beziehung, in deutscher Bearbeitung von M. 0. Fraenkel, 2. Band, Hamburg 1890

Magnan (1891): Valentin Magnan, Psychiatrische Vorlesung, Leipzig 1891

Maier (1934): Hans Wolfgang Maier, Prinzipielles zur psychiatrischen Eugenik, Schweizerische Medizinische Wochenschrift 64 (1934), Nr. 34

Maier (1908): Hans Wolfgang Maier, Über moralische Idiotie, Journal für Psychologie und Neurologie, 3 (1908), S. 57-81

Maier (1942): Hans Wolfgang Maier, Zur Frage der Sterilisation Schwachsinniger, Schweizer Erziehungsrundschau 3 (1942)

Mattner (2000): Dieter Mattner, Behinderte Menschen in der Gesellschaft. Zwischen Ausgrenzung und Integration, Stuttgart 2000

Meier/Hürlimann/Tanner (2002): Marietta Meier, Gisela Hürlimann, Jakob Tanner, Zwangsmassnahmen in der Zürcher Psychiatrie 1870-1970, Zürich 2002

Mezger (1942): Edmund Mezger, Kriminalpolitik auf kriminologischer Grundlage, Stuttgart 1942

Möbius (1901): Paul Julius Möbius, Ueber den physiologischen Schwachsinn des Weibes, Halle 1901

Moravia (1973): Sergio Moravia, Beobachtende Vernunft. Philosophie und Anthropologie in der Aufklärung, Frankfurt a. M. 1973

Morel (1857): Bénédict Augustin Morel, Traité des dégénérescences physiques, intellectuelles et morales de l'éspèce humaine, Paris 1857

Müller (2001): Bertold Müller, Rechtliche und gesellschaftliche Stellung von Menschen mit einer "geistigen Behinderung", Zürich 2001

Müller (1996): Klaus E. Müller, Der Krüppel. Ethnologia passionis humanae, München 1996

Mürner (1996): Christian Mürner, Philosophische Bedrohungen. Kommentare zur Bewertung der Behinderung, Frankfurt 1996

Nordau (1896): Max Nordau, Entartung, Berlin 1896 
Oesch (1956): Hans Oesch, Die Krankheit des Hermannus Contractus, Basel 1956

Ohler (1990): Norbert Ohler, Sterben und Tod im Mittelalter, Düsseldorf 1990

Pius XI. (1930): Papst Pius XI., Enzyklika Casti connubii, 31.12.1930, www.stjosef.at/dokumente/casti_connubii. htm

Radkau (1998): Joachim Radkau, Das Zeitalter der Nervosität, München 1998

Ritter (1889): Adolf Ritter (Hrsg.), Verhandlungen der 1. Schweizerischen Konferenz für das Idiotenwesen in Zürich am 3. und 4. Juni 1889, Zürich 1889

Ritter (2000): Hans Jakob Ritter, Nicht unbeeinflusst durch nördliche Winde? Schweizer Psychiatrie und Eugenik in der Zwischenkriegszeit, Psychiatrische Praxis 27 (2000), S. 127-133

Roelcke (1999): Volker Roelcke, Krankheit und Kulturkritik. Psychiatrische Gesellschaftsdeutungen im bürgerlichen Zeitalter (1790-1914), Frankfurt a. M. 1999

Rohrer (1945): Oswald Rohrer, Die Fürsorge für körperlich und geistig Behinderte in der Schweiz, in: Stavros Zurukzoglu (Hrsg.), Gesundheitspflege der Gegenwart, Beiheft 2, Bern 1945

Roth (1948): Agnes Roth, Ich klage an. Wahre Berichte und Selbsterlebnisse aus Irrenhäusern, Zürich 1948

Rufer (1997): Marc Rufer, Irrsinn Psychiatrie, Vollständig überarbeitete Neuauflage, Bern 1997

Sarasin (2001): Philipp Sarasin, Reizbare Maschinen. Eine Geschichte des Körpers 1765-1914, Frankfurt a. M. 2001

Sarasin (2003 a): Philipp Sarasin, Geschichtswissenschaft und Diskursanalyse, Frankfurt a. M. 2003

Sarasin (2003 b): Philipp Sarasin, Zweierlei Rassismus? Die Selektion des Fremden als Problem in Michel Foucaults Verbindung von Biopolitik und Rassismus, in: Martin Stingelin (Hrsg.), Biopolitik und Rassismus, Frankfurt a. M. 2003, S. 55-79

Schallmayer (1891): Wilhelm Schallmeyer, Ueber die drohende physische Entartung der Culturmenschheit und die Verstaatlichung des ärztlichen Standes, Berlin 1891

Schallmayer (1907): Wilhelm Schallmeyer, Vererbung und Auslese als Faktoren zu Tüchtigkeit und Entartung der Völker, Berlin 1907

Schawalder (1999): Susanne Schawalder, Flügel in Ketten. Die schweizerische Vereinigung Anormale (Pro Infirmis) und ihre Legitimation einer „Anormalenhilfe“ 1920-1955, unveröffentlichte Lizentiatsarbeit, Zürich 1999

Scheugl (1974): Hans Scheugl, Show Freaks und Monster. Sammlung Felix Adanos, Köln 1974

Schlagenhaufen (1916): Otto Schlagenhaufen, Sozialanthropologie und Krieg. Vortrag gehalten vor dem Zürcher Hochschulverein Zürich, Leipzig 1916

Schmid (1939): Werner Schmid, Jung-Schweizer! Jung-Schweizerinnen! Das Schicksal des Vaterlands ruht in Euch!, Zürich 1939

Schmutz (2000): Hans-Konrad Schmutz, Schokolade und Messzirkel - Zur Steuerung rassenhygienischer Forschungsprojekte an der Universität Zürich in den zwanziger und dreißiger Jahren. Die Tätigkeit der Julius Klaus-Stiftung für Vererbungsforschung, Sozialanthropologie und Rassenhygiene, in: Ekkehard Höxtermann, Joachim Kaasch, Michael Kaasch (Hrsg.), Berichte zur Geschichte und Theorie der Ökologie, Neuenburg a.d. Donau 2000

Schneidmüller (2000): Bernd Schneidmüller, Die Welfen. Herrschaft und Erinnerung (819-1252), Stuttgart 2000

Schulte (1997): Christoph Schulte, Psychopathologie des Fin de Siècle. Der Kulturkritiker, Arzt und Zionist Max Nordau, Frankfurt a. M. 1997

Senn (1975): Matthias Senn, Johann Jakob Wicks Nachrichtensammlung aus dem 16. Jahrhundert, Zürich 1975

Sonderegger (1927): Albert Sonderegger, Missgeburten und Wundererscheinungen in Einblattdrucken und Handzeichnungen des 16. Jahrhunderts, Band XII, in: Gustav Adolf Wehrli (Hrsg.), Zürcher Medizingeschichtliche Abhandlungen, Zürich 1927

Stingelin (2003): Martin Stingelin (Hrsg.), Biopolitik und Rassismus, Frankfurt a. M. 2003

Stolberg (2003): Michael Stolberg, The Crime of Onan and the Laws of Nature. Religious and medical discourses on masturbation in the late seventeenth and early eighteenth centuries, Paedagogica Historica, Volume 39, Issue 6, December 2003, p. 701-717 


\section{$G$ Behinderungen}

Szasz (1977): Thomas Szasz, The Theology of Medicine. The political-philosophical foundations of medical ethics, New York 1977

Tervooren (2002): Anja Tervooren, Freak-Show und Körperinszenierungen, Behindertenpädagogik 2 (2002), S. $173-184$

Tervooren (2003): Anja Tervooren, Disability Studies - vom Defizit zum Kennzeichen, Impulse. Newsletter zur Gesundheitsförderung 39 (2003), S. 17

Thürer/Aubert/Müller (2001): Daniel Thürer, Jean-François Aubert, Jörg-Paul Müller, Verfassungsrecht der Schweiz, Zürich 2001

Trus (1995): Armin Trus, „... vom Leid erlösen“. Zur Geschichte der nationalsozialistischen „Euthanasie“-Verbrechen, Frankfurt a. M. 1995

Wacke (1989): Andreas Wacke, Vom Hermaphroditen zum Transsexuellen. Zur Stellung von Zwittern in der Rechtsgeschichte, in: Eyrich, Heinz/Odersky, Walter/Säcker, Franz Jürgen (Hrsg.), Festschrift füt Kurt Rebmann zum 65. Geburtstag, München 1989, S. 861-903

Waldschmidt (2006): Anne Waldschmidt, Soziales Problem oder kulturelle Differenz? Zur Thematisierungsgeschichte von „Behinderung" aus der Sicht der Disability Studies, Traverse 3 (2006), S. 31-46

Weisser/Renggli (2004): Jan Weisser, Cornelia Renggli (Hrsg.), Disability Studies. Ein Lesebuch, Biel 2004

Westphal (1869): Carl Westphal, Die conträre Sexualempfindung, Symptom eines neuropathischen (psychopathischen) Zustandes, Archiv für Psychiatrie und Nervenkrankheiten, Bd. 1, Berlin 1869

Wolfisberg (1995): Carlo Wolfisberg, Behinderte im Spätmittelalter, Liz. Zürich 1995

Wolfisberg (2002): Carlo Wolfisberg, Heilpädagogik und Eugenik. Zur Geschichte der Heilpädagogik in der deutschsprachigen Schweiz (1800-1950), Zürich 2002

Wottreng (1999): Willi Wottreng, Hirnriss. Wie die Irrenärzte August Forel und Eugen Bleuler das Menschengeschlecht retten wollten, Zürich 1999

Wyrsch (1946): Jakob Wyrsch, Gerichtliche Psychiatrie, Bern 1946

Zürcher (2004): Urs Zürcher, Monster oder Laune der Natur, Medizin und die Lehre von Missbildungen 17801914, Frankfurt a. M. 2004

Zurukzoglu (1925): Stavros Zurukzoglu, Biologische Probleme der Rassenhygiene und die Kulturvölker, München 1925

Zurukzoglu (1938): Stavros Zurukzoglu (Hrsg.), Verhütung erbkranken Nachwuchses, Basel 1938 